\title{
The path to visualization of walking myosin $V$ by high-speed atomic force microscopy
}

\author{
Noriyuki Kodera • Toshio Ando
}

Received: 27 December 2013 / Accepted: 7 May 2014 / Published online: 18 June 2014

(C) The Author(s) 2014. This article is published with open access at Springerlink.com

\begin{abstract}
The quest for understanding the mechanism of myosin-based motility started with studies on muscle contraction. From numerous studies, the basic frameworks for this mechanism were constructed and brilliant hypotheses were put forward. However, the argument about the most crucial issue of how the actin-myosin interaction generates contractile force and shortening has not been definitive. To increase the "directness of measurement", in vitro motility assays and single-molecule optical techniques were created and used. Consequently, detailed knowledge of the motility of muscle myosin evolved, which resulted in provoking more arguments to a higher level. In parallel with technical progress, advances in cell biology led to the discovery of many classes of myosins. Myosin V was discovered to be a processive motor, unlike myosin II. The processivity reduced experimental difficulties because it allowed continuous tracing of the motor action of single myosin $\mathrm{V}$ molecules. Extensive studies of myosin $\mathrm{V}$ were expected to resolve arguments and build a consensus but did not necessarily do so. The directness of measurement was further enhanced by the recent advent of high-speed atomic force microscopy capable of directly visualizing biological molecules in action at high spatiotemporal
\end{abstract}

\footnotetext{
N. Kodera $\cdot$ T. Ando

Bio-AFM Frontier Research Center, Kanazawa University, Kanazawa 920-1192, Japan

T. Ando $(\bowtie)$

Department of Physics, College of Science and Engineering, Kanazawa University, Kakuma-machi, Kanazawa 920-1192, Japan e-mail: tando@staff.kanazawa-u.ac.jp

\section{N. Kodera}

PREST, Japan Science and Technology Agency, 4-1-8 Honcho, Kawaguchi 332-0012, Japan

T. Ando

CREST, Japan Science and Technology Agency, 4-1-8 Honcho, Kawaguchi 332-0012, Japan
}

resolution. This microscopy clearly visualized myosin $\mathrm{V}$ molecules walking on actin filaments and at last provided irrefutable evidence for the swinging lever-arm motion propelling the molecules. However, a peculiar foot stomp behavior also appeared in the AFM movie, raising new questions of the chemo-mechanical coupling in this motor and myosin motors in general. This article reviews these changes in the research of myosin motility and proposes new ideas to resolve the newly raised questions.

Keywords Myosin $\cdot$ Actin $\cdot$ Muscle $\cdot$ Motor proteins · High-speed AFM $\cdot$ Imaging

\section{Introduction}

Motions in biological systems are vital phenomena. They inspire our curiosity to understand how motion is made possible. Skeletal muscles exhibit striking physiological action (i.e. contraction) that is visible to the naked eye and is daily experienced by ourselves. It was, therefore, natural that research into biological motility started with the analysis of muscles. Biochemical studies of muscle proteins revealed that the molecular entities responsible for generating contractile force and shortening comprise just three: myosin, actin, and ATP. Each head of the double-headed myosin hydrolyzes ATP into ADP and inorganic phosphate (Pi). The ATPase rate is very low when myosin is alone but is markedly accelerated by its interaction with actin, where the chemical energy liberated by ATP hydrolysis is converted into mechanical work. This energy conversion has most attracted our curiosity and thus been extensively studied using physiological, biophysical, biochemical, structural biological, and theoretical methods. It is noteworthy that several experimental techniques have been applied or created to study muscle contraction, and later used in other fields of biological science. Results obtained in 
each discipline have built important frameworks and hypotheses for the mechanism of muscle contraction. For example: (1) the relationship between load, speed of shortening, and the rate of heat production in muscle contraction (Hill 1938, 1964); (2) the filament sliding mechanism in which muscle shortens as a result of the mutual sliding of thin filaments containing actin relative to thick filaments containing myosin (Huxley 1953; Huxley and Hanson 1954; Huxley and Niedergerke 1954); (3) the generation of force by myosin cross-bridges that extend from the myosin filaments to the adjacent actin filaments in the overlap zone in the sarcomere (Huxley 1957a, b); (4) the hypothesis of swinging lever-arm as powerstroke (Huxley 1969); (5) the concept of a working stroke of $\sim 10 \mathrm{~nm}$ derived from the swinging lever-arm hypothesis and from the observation of tension recovery following a quick release applied to contracting muscle fibers (Huxley and Simmons 1971); and (6) the kinetic scheme of the actomyosin ATPase reaction (Lymn and Taylor 1971). These ideas were integrated into a general consensus on the muscle contraction mechanism. Nonetheless, a clue to greater clarification of the chemo-mechanical reaction in actomyosin unexpectedly emerged from the discovery of non-muscle myosins together with the creation of single-molecule biophysical techniques.

Currently, there are 35 (or more) distinct classes of myosins designated I to XXXV (Berg et al. 2001; Thompson and Langford 2002; Richards and Cavalier-Smith 2005; Foth et al. 2006; Odronitz and Kollmar 2007). They constitute the superfamily of actin-based motors that play crucial roles in dynamic cellular processes. In the initial attempt to find nonmuscle myosins, first a single-headed myosin (Pollard and Korn 1973) and subsequently a traditional double-headed myosin (Maruta and Korn 1977) were isolated from Acanthamoeba. Later, the former was called "myosin I" and the latter "myosin II". Thereafter, the Roman numerals have been given according to the chronological order of discovery. Among the 35 classes of myosins, myosin V (Mehta et al. 1999; Sakamoto et al. 2000), VI (Rock et al. 2001; Nishikawa et al. 2002), VII (Yang et al. 2006), X (Nagy et al. 2008; Ricca and Rock 2010; Sun et al. 2010), and XI (Tominaga et al. 2003; Hachikubo et al. 2007) are known to be processive motors, i.e. a single molecule undergoes multiple catalytic cycles and mechanical advances before dissociation from an actin filament. This processivity promoted the study of myosin motility because it enabled us to continuously trace the motor action of individual molecules. Note that not all isoforms in each of these classes are processive. For example, the yeast myosin V, Myo2p (Reck-Peterson et al. 2001), and the Chara myosin XI (Kimura et al. 2003) are not processive, although Myo2p is switched to a processive motor when tropomyosin is bound to actin filaments (Hodges et al. 2012).

In parallel with the progress of non-muscle myosin studies, fluorescence microscopy (see review by Joo et al. 2008) and optical trap nanometry (see review by Neuman and Block 2004) were developed to observe the dynamic action of proteins at the single molecule level. These techniques assisted the discovery of several properties of myosin $\mathrm{V}$ (hereafter referred to as M5) including three-to-five successive step advances under load with the $\sim 36-\mathrm{nm}$ step size (Mehta et al. 1999), processive runs of several micrometers under no load (Sakamoto et al. 2000), load-dependent stepping kinetics (Veigel et al. 2002), the hand-over-hand manner of this movement (Forkey et al. 2003; Yildiz et al. 2003), and the tight relationship between the catalytic cycle and the $36-\mathrm{nm}$ advance (Sakamoto et al. 2008). These discoveries led to a comprehensive view for the action of myosin motors. Nonetheless, a complete consensus has not been reached and more details of the motor action and the underlying chemomechanical coupling have remained elusive.

Looking back on the long history of myosin motility studies, we notice that the gap between experimental data and conclusions derived therefrom has been slowly reducing. This is because the level of "directness of measurement" has been increasing. Before the single-molecule era, we had to infer the action of individual molecules from experimental data ensemble-averaged over many molecules. Several details of the action of myosin were hidden in the ensemble-average measurements on bulk samples. However, even when singlemolecules were observed, their level of directness was not perfect because the subset of observed molecular events depends on the site where an optical probe is placed while the entire molecular structure remained invisible. Myosin has been studied by X-ray crystallography, NMR, and electron microscopy, but these structures are limited to static snapshots and the simultaneous assessment of structure and dynamics seemed impossible.

High-speed atomic force microscopy (HS-AFM), which started to be developed around 1993 to overcome this limitation, is now sufficiently advanced (Ando et al. 2008) to allow us to video-record the structure and dynamics of functioning biomolecules at single-nanometer resolution, without disturbing their function. This new microscopy was applied to M5 (Kodera et al. 2010). The obtained image data revealed details of its molecular action, leading not only to the corroboration of known or inferred behavior of the motor but also to new discoveries (Kodera et al. 2010). In particular, it was a surprise to discover that the tension responsible for forward movement can be generated without transitioning through an ADP-Pi-bound state, meaning that no chemical energy input is required for the tension generation. Moreover, the lever-arm swing (powerstroke) by the leading head spontaneously occurs when the trailing head detaches, thus demonstrating that no chemical energy input is required for the lever-arm swing either. These findings appear inconsistent with a popular scheme put forward for the chemo-mechanical reaction in muscle contraction (e.g., Geeves and Holmes 1999; Goldman 
2004). Thus, we now need to develop a unified motor mechanism that can be applied to all classes of myosin motors.

This review does not cover the entire range of myosin motors but focuses on studies of the motor mechanism, particularly those performed with the use of single-molecule techniques and our recent HS-AFM study of M5 motility. Readers are referred to reviews on muscle contraction (Geeves and Holmes 1999; Huxley 2000, 2004; Goldman 2004), on structure-function relationships of myosins (Ruppel and Spudich 1996; Sweeney and Houdusse 2010a), and on the cellular functions of various myosins (Hartman et al. 2011; Coluccio 2008).

\section{General aspects of myosin}

The myosin superfamily is largely categorized into the monomeric and dimeric types (Pecham 2011), although some myosins can adopt both forms depending on the environment (Park et al. 2006; Yu et al. 2009; Tokuo et al. 2007; Umeki et al. 2011) and both types of isoforms sometimes exist in the same class (e.g., monomeric yeast Myo4p and dimeric yeast Myo2p belonging to class V; Bookwalter et al. 2009). In stable dimeric myosins, the N-terminal head is followed by a coiledcoil tail domain for dimerization, while in monomeric myosins it is followed by a tail domain or a stable, single $\alpha$-helical (SAH) domain (Knight et al. 2005) and then by a tail domain. The C-terminal tail end generally serves as a cargo-binding domain (Fig. 1). In this regard, dimeric myosin II is rather unique. It does not possess a cargo-binding domain and assembles into bipolar thick filaments mediated by a long coiled-coil tail. The tail and cargo-binding domains have diverse amino acid sequences, depending on the intracellular function of myosin (e.g., Sellers 2000), while the head region comprising a motor domain and a neck domain is generally common. The motor domain contains two functional sites, the actin-binding and nucleotide-binding sites which communicate with each other. The neck domain comprises a variable number of IQ motifs that bind calmodulin or myosin light chains. The neck is connected to the so-called converter region in the head, and these two as a single entity is called the leverarm. When the SAH domain follows the neck, it is also included in the lever-arm because it functionally extends the length of the canonical lever-arm (Baboolal et al. 2009). The heavy chain in the head comprises three parts $(25,50$, and 20 $23 \mathrm{kDa}$ ) aligned in this order from the $\mathrm{N}$-terminus that were revealed by limited tryptic cleavage of subfragment-1 (S-1) of skeletal myosin II (Mornet et al. 1979; Mornet et al. 1981). These three parts connected to two surface loops are arranged as shown in Fig. 2 in the three-dimensional (3D) structure of the head (Rayment et al. 1993a; Houdusse et al. 2000). This $3 \mathrm{D}$ arrangement of the three parts is largely common to all classes of myosin. The most prominent feature is the presence of a large cleft dividing the $50-\mathrm{K}$ subdomain into lower and upper $50-\mathrm{K}$ subdomains. The outer cleft regions of both lower and upper $50-\mathrm{K}$ subdomains bind to actin mainly through loops. The ATP-binding site is located at the inner cleft region, around which all the three parts face each other. Interestingly, the 3D structure of the catalytic core is very similar to the entire motor domain structure of kinesin despite complete dissimilarity in the amino acid sequence, suggesting that they share a similar force-generating strategy (Kull et al. 1996).

Single-headed myosin S-1 binds to actin at a given angle relative to the actin filament. The bound single head (both motor and neck domains) often extends towards the plus end of actin at least in the nucleotide-free and ADPbound conditions (Rayment et al. 1993b; Jontes et al. 1995). This orientation towards the plus end of actin is called "arrowhead orientation" (Huxley 1963). Among all members of the myosin superfamily, only myosin VI is known to move towards the minus (barbed) end of actin (Wells et al. 1999). The motor domain of a single-headed myosin VI construct also binds to actin in the arrowhead orientation. However, its neck domain is largely kinked relative to the motor domain, so that the neck domain orients slightly towards the minus end of actin (Wells et al. 1999). The swinging lever-arm hypothesis presumes that the lever-arm rotates so that a minute change in the motor domain is amplified to a large displacement of the lever-arm's distal end. When this presumption is applied to myosin VI, its reversal movement must be achieved by the rotation of the lever-arm in the direction opposite to that of the other myosins (Wells et al. 1999). This is consistent with the kinked neck domain of myosin VI and also with the reversal movement of a myosin I construct with an artificial neck which is kinked towards the reversearrowhead orientation (Tsiavaliaris et al. 2004).

The actomyosin ATPase reaction proceeds as shown in Fig. 3. Immediately after binding to ATP, the actin-bound head detaches from actin, followed by quick hydrolysis of the bound ATP to ADP-Pi. The ADP-Pi-bound head has a low affinity for actin (yet a somewhat stronger affinity than the ATP-bound head). When the ADP-Pi-bound head is attached to actin, the bound Pi dissociates from the head, followed by the formation of a strongly-bound complex of A-M-ADP (A and $\mathrm{M}$ denote actin and myosin head, respectively), and then by ADP dissociation. The nucleotide-free head also has a strong affinity for actin. These complexes with strong affinities are called "rigor complexes". Thus, a myosin head has two states with respect to its affinity for actin, the weak- and strong-binding states. The main role of actin in the ATPase reaction is to accelerate the otherwise very slow Pi and ADP dissociation from a myosin head. The degree of acceleration for Pi dissociation and ADP dissociation varies among the classes of myosin. The processivity occurs in dymeric 


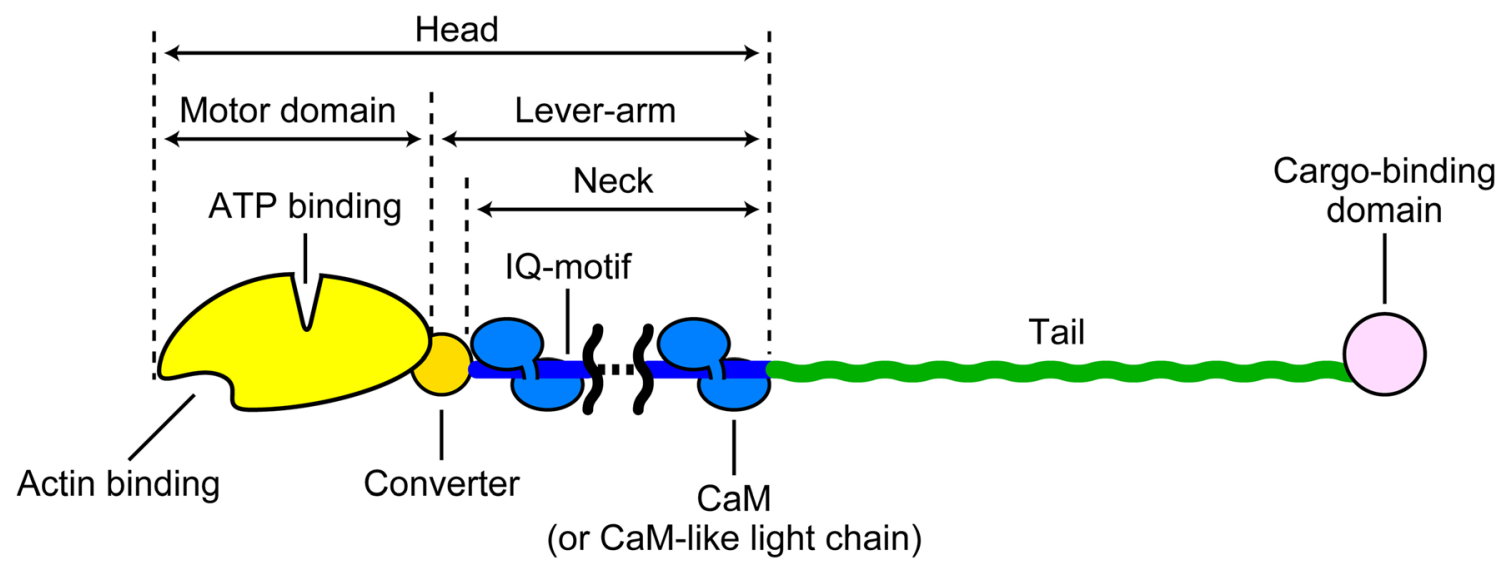

Fig. 1 Schematic showing domain organization of myosin molecule. The tail part is variable and contains an $\alpha$-helical coiled-coil, a SAH domain, or a chain segment with a variable amino-acid sequence,

myosins when the lifetime of A-M-ADP predominates over the other states (De La Cruz et al. 1999).

In the prevailing view on myosin motility, the chemomechanical coupling is considered to occur as follows (see Fig. 3). The myosin head undergoes conformational changes upon ATP binding and hydrolysis to ADP-Pi. Myosin then swings back the lever-arm ("recovery stroke") (Geeves and Holmes 1999). The angle change between the motor and neck domains caused by this recovery stroke facilitates the binding of the head to actin in the reverse arrowhead orientation (note that the motor domain still binds to actin in the arrowhead orientation). The A-M-ADP-Pi state, often termed as the "pre-powerstroke state", is considered to be a higher-energy state. Upon Pi release, tension for driving the lever-arm swing is generated, the lever-arm actually swings to execute a powerstroke, and then a lower-energy state of A-M-ADP (the post-powerstroke state) is formed. Thus, in this view, the chemical energy liberated by ATP hydrolysis is used mainly in the Pi-release step. depending on the myosin class or isoform. When a SAH domain follows the neck, it is included in the lever-arm because it functionally extends the canonical lever-arm

\section{Progress of motility assays}

The situation before the single-molecule era

Almost the entire enzymatic kinetics of actomyosin has been obtained from muscle myosin. In most of kinetics measurements, soluble forms of myosin, S-1 and heavy meromyosin (HMM), were used to simplify interpretations of the data. Then, the enzymatic kinetics and the structural characteristics of myosin were combined to understand how the myosin motor might work. Although the overall feature of the enzymatic kinetics was successfully revealed, no mechanical constraints were imposed to myosin molecules interacting with actin filaments in vitro. Therefore, it was difficult to deduce the most important characteristic of the mechano-enzyme, namely how does mechanical strain imposed on myosin affect the ATPase reaction? Moreover, the whole cycle of the ATPase reaction could not be tracked down in one measurement because the initial synchrony among molecules becomes
Fig. 2 Crystal structure of skeletal myosin II S-1. The heavy chain of myosin is shown as a ribbon diagram, while two light chains [i.e. essential light chain $(E L C)$ and regulatory light chain $(R L C)]$ are shown as a sphere diagram. The 25-, 50-, and 20$\mathrm{kDa}$ regions are colored green, red, and blue, respectively. The converter domain in the $20-\mathrm{kDa}$ region is additionally colored with orange, after which IQ motifs follow

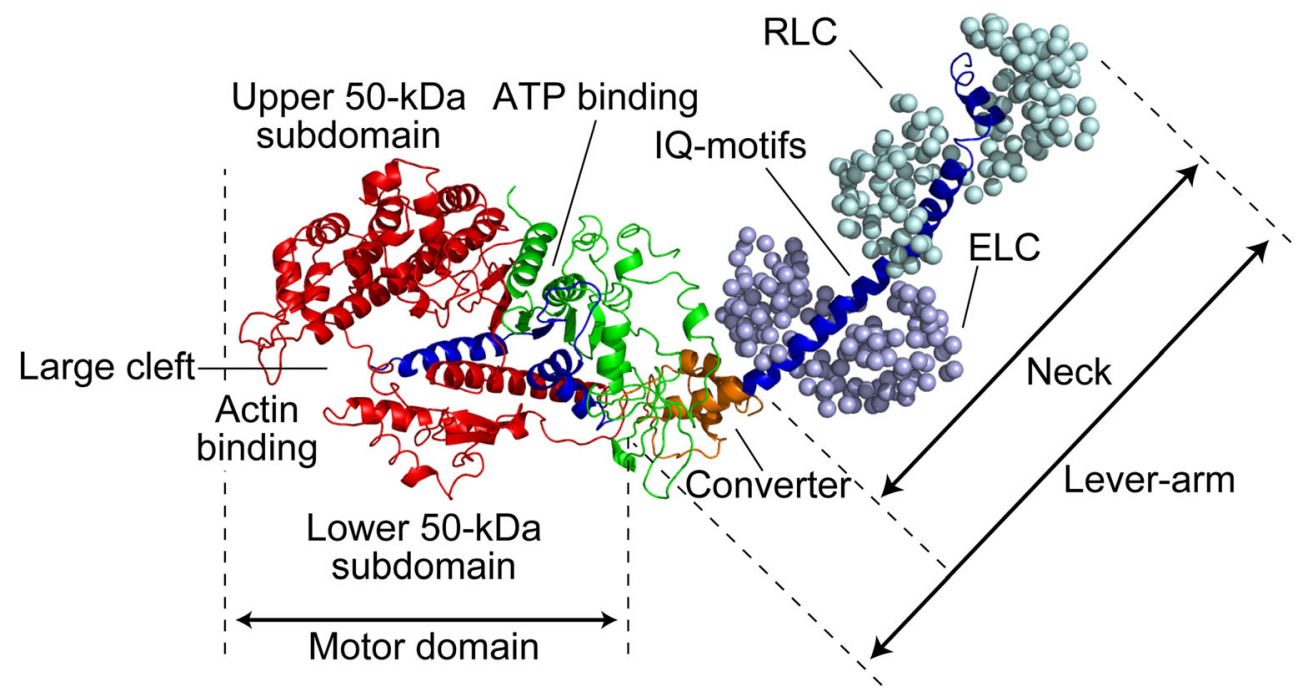




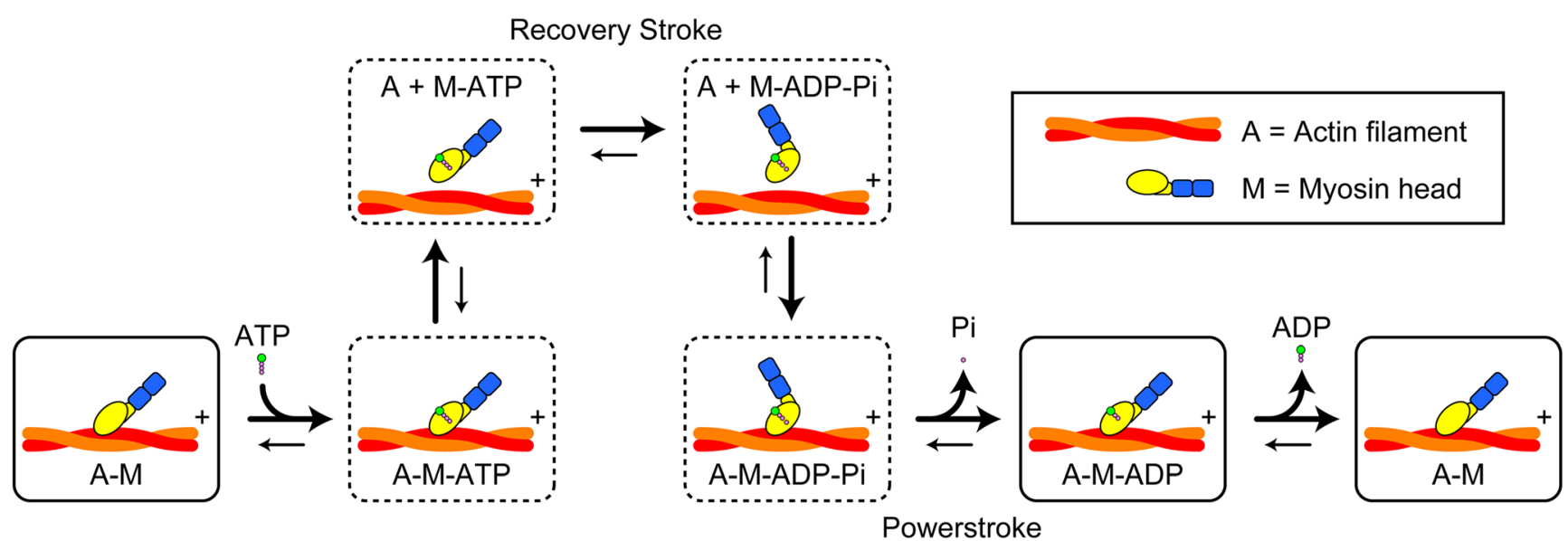

Fig. 3 Scheme of muscle actomyosin ATPase reaction including structural model. + indicates the plus end of actin. Boxes with solid lines and broken lines represent the strong- and weak-affinity states, respectively. The powerstroke is considered to occur mainly in the transition from the

obscured by the stochastic nature of the reaction. Therefore, many kinetic constants derived for the entire reaction scheme were obtained in separate experiments where the reactions were started with different initial states. In some cases, it was difficult to prepare adequate initial states. As such, a quantitative reaction scheme was obtained by "pasting" many results together that were not necessarily obtained under similar solution conditions.

The mechanical behavior of myosin interacting with actin filaments could not be observed in solution experiments. To acquire this information, muscle fibers and myofibrils were subjected to X-ray diffraction (Huxley et al. 1981, 1983), fluorescence polarization (Nihei et al. 1974; Borejdo and Putnam 1977), electron paramagnetic resonance (EPR) spectroscopy (Thomas and Cooke 1980), and force analyses (Ford et al. 1977). At a very early stage intrinsic tryptophan fluorescence or fluorescent ATP analogues were used for fluorescence polarization studies (dos Remedios et al. 1972a, b). Later, fluorescence and spin polarization probes were introduced to a specific cysteine, SH1, in the motor domain. This was possible because SH1 is most reactive of all myosin cysteines (Sekine and Kielly 1964) and indeed of all proteins in skinned muscle fibers (Borejdo and Putnam 1977), and therefore could be selectively labeled. Unfortunately, it later became known that the SH1-labeled myosin does not function as a motor (Root and Reisler 1992; Marriott and Heidecker 1996; Bobkov et al. 1997). Extensive muscle fiber X-ray diffraction studies were performed but no clear evidence for cross-bridge movement was obtained. In the light of numerous spectroscopic and structural studies, the massive part of an actin-bound myosin head proximal to actin was suggested to remain unchanged during the powerstroke, whereas the distal neck region with smaller mass than the proximal one was suggested to change its orientation (Cooke 1986). In this case it would be difficult to distinctly detect this change in the fiber
A-M-ADP-Pi state to the A-M-ADP state. The recovery stroke is considered to occur in the transition from the M-ATP state to the MADP-Pi state

diffraction patterns. Moreover, the time in which a myosin head interacts with an actin is very short in the ATPase cycle, making it difficult to record the dynamics of cross-bridges.

The molecular structures of myosin heads in different nucleotide conditions were successfully revealed at submolecular level using electron microscopy (e.g., Walker and Trinick 1988; Katayama 1989; Pollard et al. 1993; Takács et al. 2010) and at the atomic level using X-ray crystallography (e.g., Rayment et al. 1993a; Fisher et al. 1995; Dominguez et al. 1998; Houdusse et al. 2000). However, as a matter of course, these structures were static snapshots. Moreover, even with the current state of technology, it is still difficult to obtain the atomic structure of actin-bound myosin head, particularly the structure in the power-stroking state (Rayment et al. 1993b; Lorenz and Holmes 2010).

\section{In vitro motility assays}

To overcome some of the above adversities, experimental techniques called in vitro motility assays were developed in the early 1980s, as advanced versions of previous methods developed in the 1970s (Oplatka and Tirosh 1973; Kuroda and Kamiya 1975). Dark-field microscopy was performed that detected the movement of fibrils that were squeezed out of the giant alga Nitella cell, washed with buffer, and mixed with ATP (Higashi-Fujime 1980). Next, fluorescence microscopy was conducted using a more purified system where skeletal myosin-coated fluorescent beads were observed to slide on a substratum of polar arrays of actin cables derived from giant alga, Nitella (Fig. 4a) (Sheetz and Spudich 1983). The movement was ATP-dependent and blocked by inactivation of the myosin heads using N-ethylmaleimide. This study revealed that the bipolar thick filament structure is not necessary for generating force and movement. Remarkably, this assay system was much simpler and more robust than previous in vitro 
motility assay systems (Oplatka and Tirosh 1973; Kuroda and Kamiya 1975; Higashi-Fujime 1980; Yano et al. 1982), and this facilitated quantitative analyses of the myosin motility. Using fluorescently labeled single actin filaments, their thermal bending motion was observed to increase in the presence of HMM and ATP (Yanagida et al. 1984). Also was demonstrated that the sliding movement of individual actin filaments on myosin-coated surfaces occurred at velocities similar to those recorded in muscles (Fig. 4b) (Spudich et al. 1985). Moreover, actin filaments were observed to slide on oneheaded myosin filaments, indicating that a two-headed structure is not needed for motility (Harada et al. 1987). This result was reinforced by the observation of actin filaments gliding over myosin S1-coated surfaces (Toyoshima et al. 1987). Thus, motor activity inherent in the head was established, which ruled out some models of muscle contraction.

\section{In vitro motility studies on chemo-mechanical relationship}

Understanding how actomyosin ATPase kinetics determines the mechanical performance of this motor system (magnitude of generated force and sliding velocity) became a central focus of muscle research. Even in the in vitro motility assays, single actin filament sliding and the translational motion of myosincoated beads are propelled by multiple myosin heads. Therefore, the chemo-mechanical relationship was not straightforward. Nonetheless, the in vitro motility assays made the analysis more precise and more extensive than previous assays where muscle fibers were studied. The in vitro motility assays allowed us to investigate actomyosin motility under wide varieties of solution conditions with myosins and actins from diverse species and cell types. The velocity of actin filament sliding over myosin-coated surfaces and the velocity of myosin-coated beads along actin filaments were shown to be analogous to the speed of unloaded shortening of muscle fibers (Sheetz et al. 1984; Spudich et al. 1985; Kron and Spudich 1986; Homsher et al. 1992). Despite the reported analogy, many studies using different types of myosin II found no direct correlation between the steady-state actomyosin ATPase activity and the rate of movement measured by the in vitro motility assays (Umemoto et al. 1989; Lowey et al. 1993; Uyeda et al. 1994; Vale et al. 1984; Higashi-Fujime 1991). Nor was direct correlation observed using different nucleotide triphosphates (NTPs) condition (Shimizu et al. 1991; Higashi-Fujime and Hozumi 1996). In these studies, one concentration of actin and one concentration of NTPs were used in the measurements of the enzymatic activities. In similar studies where the actin concentration was varied and a fixed concentration of NTPs was used, a moderate correlation was shown between the motor activity and the substrate turnover rate (Pate et al. 1993a; Regnier et al. 1998).
A more extensive study was conducted for rabbit skeletal acto-HMM using six naturally occurring NTPs with $\mathrm{Mg}^{2+}$, different divalent cations with ATP, and $\mathrm{Mg}^{2+}$-ATP at various ionic strengths (Amitani et al. 2001). The maximum substrate turnover rates $\left(V_{\max }\right), K_{\mathrm{m}}$ for actin $\left(K_{\mathrm{m}}^{\mathrm{A}}\right)$, and $K_{\mathrm{m}}$ for metal ${ }^{2+}$ NTPs $\left(K_{\mathrm{m}}^{\mathrm{N}}\right)$ were obtained while the velocity of actin filament gliding on HMM-coated surfaces was measured under the same buffer and substrate conditions used for the measurements of substrate turnover rate. A large dataset acquired revealed a direct linear relationship, except when different divalent cations were used with ATP. The maximum sliding velocity $\left(V_{\mathrm{s}}^{\max }\right)$ extrapolated to the infinite concentration of substrates was directly proportional to $\left(V_{\max } K_{\mathrm{m}}^{\mathrm{A}}\right)^{1 / 2}$ (Fig. 5). This experimental correlation was analyzed based on a forcebalance model, where the average sliding force (active force) produced by a productive head, $\left\langle f_{\mathrm{s}}\right\rangle$, is balanced with the average resistive force produced by an actin-bound nonproductive head, $\left\langle f_{1}\right\rangle$, which is directly proportional to the gliding velocity of the actin filament. At infinite substrate concentration, $\left\langle f_{\mathrm{s}}>\right.$ equals $p T_{\mathrm{p}} / T_{\mathrm{c}}$, where $p$ is the magnitude of powerstroke (force spike), $T_{\mathrm{p}}$ is the duration of single powerstroke, and $T_{\mathrm{c}}$ is the substrate turnover time. The power stroke speed $\left(d / T_{\mathrm{p}}\right.$; where $d$ is actin filament displacement by each powerstroke) should equal the maximum sliding velocity $\left(V_{\mathrm{s}}^{\max }\right)$ (Uyeda et al. 1991). Thus, the following relationship is derived:

$<f_{\mathrm{s}}>=p d V_{\max } / V_{\mathrm{s}}^{\max }$.

At infinite substrate concentration, the resistive force averaged over the substrate turnover time $\left\langle f_{\mathrm{r}}>\right.$ is expressed as:

$<f_{\mathrm{r}}>=\frac{1}{2} \lambda \Gamma V_{\mathrm{s}}^{\max } / k_{-}$

where $\lambda$ is the fraction of the actin-bound non-productive state over all states, $\Gamma$ is an elastic constant of the resistive head, and $k_{-}$is the dissociation rate of the resistive head. Supposing that the resistive state is the weakly bound state, $k_{-}$approximately equals $k_{+} K_{\mathrm{m}}^{\mathrm{A}}$, where $k_{+}$represents the rate constant in which a detached head in the weak-binding state binds to actin. Thus, from Eqs. (1) to (2), the following relationship is derived:

$V_{\mathrm{s}}^{\max }=\left(2 k_{+} p d K_{\mathrm{m}}^{\mathrm{A}} V_{\max } / \lambda \Gamma\right)^{1 / 2}$.

As the fraction of the resistive state $\lambda$ is likely to be proportional to $k_{+}, V_{\mathrm{s}}^{\max }$ becomes directly proportional to $\left(V_{\max } K_{\mathrm{m}}^{\mathrm{A}}\right)^{1 / 2}$, which agrees well with the experimentally observed relationship (Fig. 5). The lifetime of the weakly bound state is very short but this state occurs many times within a 
Fig. 4 Schematics showing in vitro motility assay systems for actomyosin. a Myosin-coated bead assay. The myosin-coated fluorescent beads are subjected to the polar arrays of actin cables naturally formed on chloroplastd of the alga Nitella, and movement of the beads are observed under a florescent microscope. b Actin filament gliding assay. Myosin molecules are attached to the surface of a nitrocellulose-coated coverslip and gliding motion of the fluorescently labeled actin filaments are observed under a fluorescence microscope

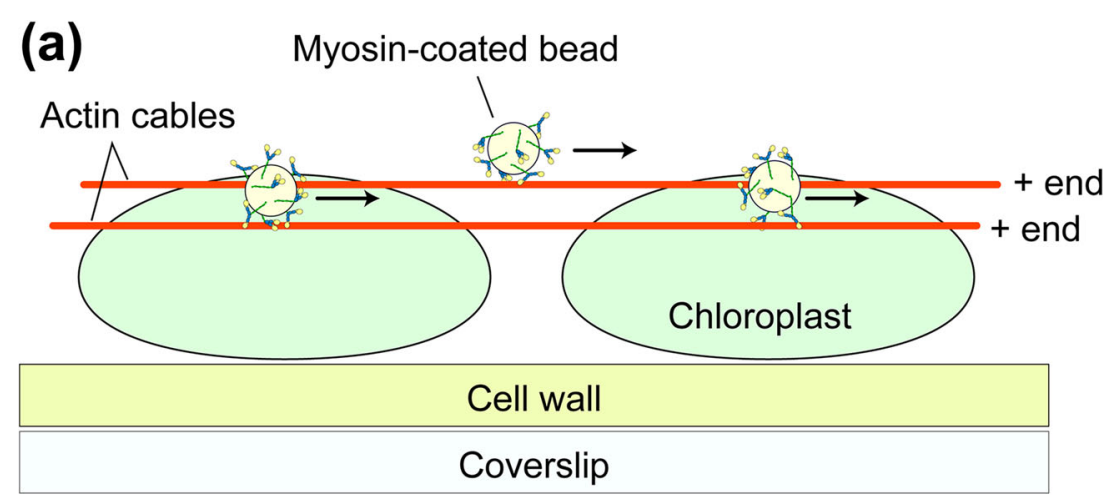

(b)

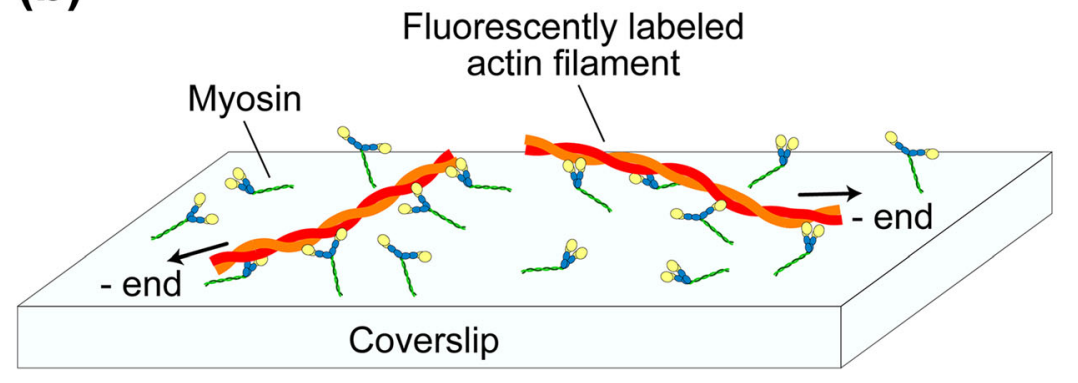

substrate turnover cycle. Thus, the weakly bound state becomes a predominant resistive state. This force balance model could also be extended to the case where actin filaments moved at various substrate concentrations by including the resistive force produced by rigor heads; thus, it could derive the dependence of sliding velocity on the substrate concentration. Its derivation showed that the dependence of the sliding velocity on the substrate concentration [S] followed a modified Michaelis-Menten equation of $V_{\mathrm{s}}=V_{\mathrm{s}}^{\max } /\left\{1+\left(K_{\mathrm{m}}^{\mathrm{N}} /[\mathrm{S}]\right)^{\mathrm{n}}\right\}$ (here, $1<\mathrm{n}<2$ ), which again agreed well with the

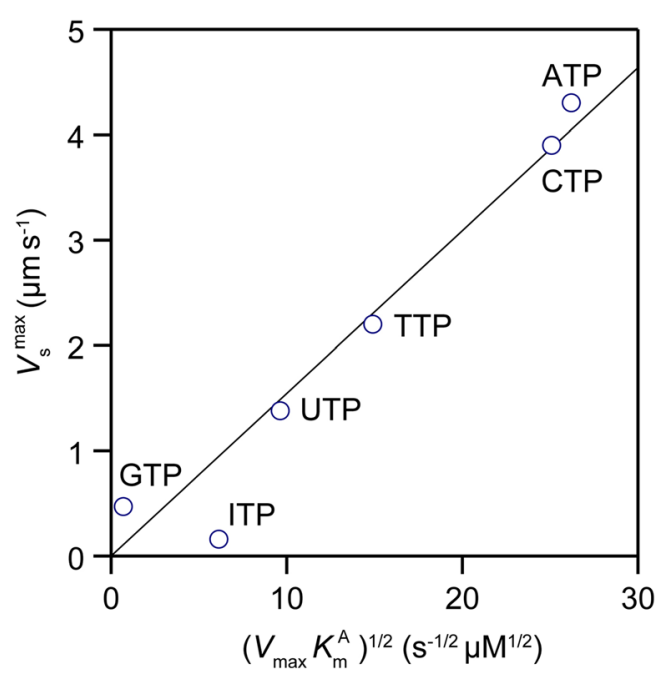

Fig. 5 Relationships between the maximum sliding velocity $\left(V_{\mathrm{s}}^{\max }\right)$ and the actomyosin NTPase kinetics parameters, $K_{\mathrm{m}}$ for actin (i.e. $K_{\mathrm{m}}^{\mathrm{A}}$ ) and maximum NTPase rate $V_{\max }$. The nucleotide substrates used are indicated by the side of the corresponding data plots experimentally obtained dependence under various conditions (Amitani et al. 2001).

Step size

The next fundamental question to tackle experimentally was the translocation distance that is propelled by actinmyosin interaction during an ATPase cycle (i.e. step size). The swinging lever-arm hypothesis predicts the size to be $\sim 10 \mathrm{~nm}$. However, as mentioned above, the in vitro actin gliding or myosin-bead gliding assay is not a singlemolecule assay. Moreover, the duration of the working stroke that occurs in an ATPase cycle has to be measured to estimate the step size but cannot be measured directly. Consequently, various values were reported for the step size: $\sim 8 \mathrm{~nm}$ (Toyoshima et al. 1990), $\sim 10 \mathrm{~nm}$ (Pate et al. 1993b), 10-28 nm (Uyeda et al. 1991), and >60 nm (Yanagida et al. 1985; Harada et al. 1990). To resolve this large discrepancy, the step size had to be directly measured at nanometer and millisecond spatiotemporal resolution. This requirement gave birth to the so-called "single-molecule measurement assays" capable of observing and manipulating single biological molecules in action.

Single-molecule measurements

Optical tweezers are typically used in single molecule measurements. A micrometer-sized dielectric particle such as a polystyrene bead is trapped by a focused laser beam (Ashkin and Dziedzic 1985; Neuman and Block 
2004) and a trapped particle can be freely moved by changing the focal position of the laser beam. The position of the trapped particle in the XY plane perpendicular to the laser beam can be measured with $\pm<1 \mathrm{~nm}$ precision. For the trapped particle, the focused laser beam works as a $3 \mathrm{D}$ spring with a spring constant of $0.01-$ $10 \mathrm{pN} / \mathrm{nm}$. When the particle is displaced by an external force in the XY plane, the magnitude of the external force applied can therefore be measured at high precision. This optical trap nanometry is now routinely used to study biological molecules (Svoboda and Block 1994; Mehta et al. 1998; Wang 1999; Bustamante et al. 2000; Kuo 2001). Step size and the force produced by a single myosin head were directly measured using a dual-beam laser trap (Finer et al. 1994; Molloy et al. 1995; Guilford et al. 1997; Tanaka et al. 1998; Veigel et al. 1999). Each end of an actin filament was attached to a particle. The two particles are caught in the dual-beam laser trap, and then the actin filament is allowed to interact with a single myosin molecule immobilized on a surface (Fig. 6a). The measured step size ranged between 3.5 and $15 \mathrm{~nm}$ but the extent of discrepancy between laboratories was much smaller than previous estimates made using actin gliding assays. It was pointed out by Yanagida's group that the measured step size tended to be affected by the interaction angle between myosin and actin. With careful design, the measured step size was shown to be dependent on the interaction angle (Tanaka et al. 1998). When an actin filament was aligned parallel with a myosin filament with a low myosin head density, a relatively large step size of $10-15 \mathrm{~nm}$ was obtained (Tanaka et al. 1998).

A very fine glass micro-needle was also used by Yanagida's group as a force transducer for single molecule mechanical measurements. A single actin filament was attached to the free end of a micro-needle and brought into contact with an immobilized single myosin molecule. The displacement of the micro-needle caused by the myosinactin interaction was measured (Kishino and Yanagida 1988; Ishijima et al. 1991; Ishijima et al. 1996). Using a low needle stiffness $\left(0.09 \mathrm{pN} \mathrm{nm}^{-1}\right)$ and near zero load, the average of single displacement spikes was $\sim 20 \mathrm{~nm}$ (Ishijima et al. 1996). However, the spatiotemporal resolution was not high enough to precisely detect the displacement spikes. Then, an experimental system was further developed (Fig. 6b); a $\mathrm{ZnO}$ crystal whisker with a tip radius of $\sim 15 \mathrm{~nm}$ was attached to a fine glass needle mounted on a three-dimensional piezoelectric actuator. A single myosin S-1 fluorescently labeled at the regulatory light chain was attached to the end of the $\mathrm{ZnO}$ whisker. The molecule was brought into contact with actin bundles formed by chicken gizzard $\alpha$-actinin, where the actin filaments were aligned in anti-parallel (Meyer and Aebi 1990). Using a glass needle with very small stiffness $\left(0.01-0.03 \mathrm{pN} \mathrm{nm}^{-1}\right)$, the spatial resolution of $2.0 \mathrm{~nm}$ and the temporal resolution of $<0.2 \mathrm{~ms}$ were obtained (Kitamura et al. 1999, 2005). Using this technique, an average step size of $\sim 13 \mathrm{~nm}$ was measured, but surprisingly several (2-5) 5.5-nm successive substeps were also detected within each step. This size, $5.5 \mathrm{~nm}$, coincides with the distance between adjacent actin monomers in a filament. More surprisingly, the dwell between substeps was independent of the ATP concentration (0.1 and $1 \mu \mathrm{M})$. Therefore, it was concluded that the multiple substeps produced by a single myosin head occurred during just one cycle of ATP hydrolysis. This conclusion was reinforced by theoretical studies (Terada et al. 2002; Takano et al. 2010). However, the experimental result has never been reported by other groups.

Total internal reflection fluorescence microscopy (TIRFM) has also been used for single-molecule measurements (Park et al. 2007; Murcia et al. 2007; Joo et al. 2008). When an incident laser light is totally reflected at the glasswater interface, an evanescent field is generated that decays exponentially from the interface, penetrating to a depth of $\sim 100 \mathrm{~nm}$ into the solution. Since only fluorophores located in the evanescent field are excited, the background light level is very low, allowing the visualization of single fluorophores (Fig. 6c). Using a Cy3-labeled ATP, the dynamic events of its binding to and dissociation from single myosin S-1 molecules were visualized (Funatsu et al. 1995). The dissociation rate estimated from the lifetime of the bound $\mathrm{Cy} 3$-nucleotide agreed with the biochemically measured rate of Cy3-ATP turnover by S-1. This success opened up a new opportunity to simultaneously measure the chemical and mechanical events in single myosin molecules. Using one-headed skeletal muscle myosin assembled with myosin rod and a setup where TIRFM and a dualbeam laser trap were combined, mechanical events of actin-myosin dissociation/force generation (displacement) and chemical events of binding/dissociation of Cy3-ATP (or Cy3-ADP) were simultaneously observed (Ishijima et al. 1998). The timing of Cy3-ATP binding well coincided with the timing of myosin head dissociation from actin. However, in more than $50 \%$ of 85 detected events, the actin filament was displaced $0.3-1.8 \mathrm{~s}$ after the dissociation of Cy3-nucleotide from the myosin head. This surprising result suggested that myosin has a hysteresis or memory state in which the chemical energy liberated by ATP hydrolysis is stored even after ADP dissociation. However, photobleaching of bound Cy3-nucleotide also looks like it is dissociated from the myosin head. Therefore, their interpretation could not be statistically verified. The step size measured in this study $(\sim 15 \mathrm{~nm})$ was consistent with their previously reported values.

Single-molecule measurements quantified other mechanical properties of actin, myosin, and their interactions. The tensile 
(a)

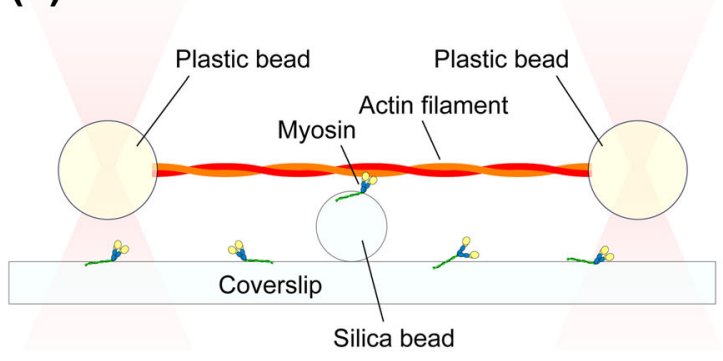

(b)

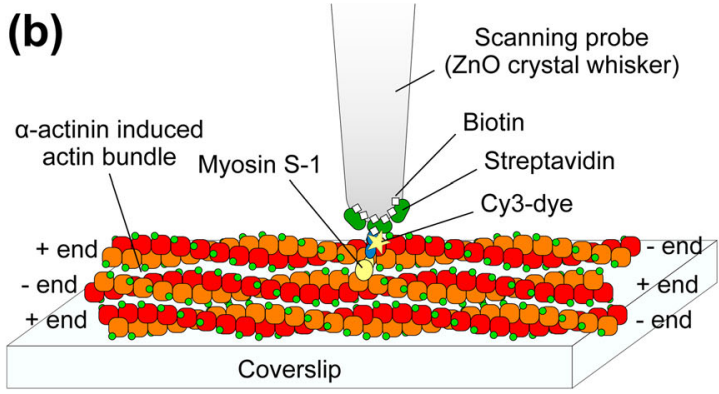

(c)

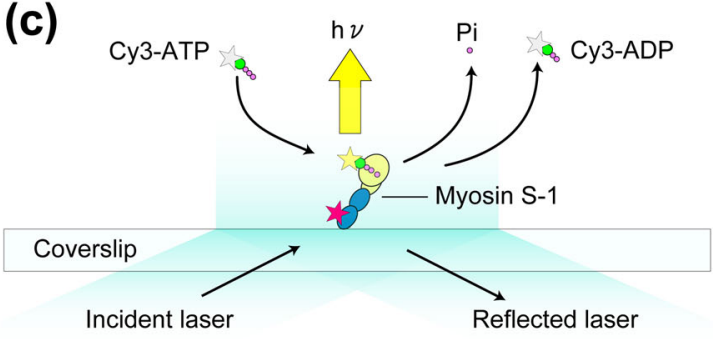

Fig. 6 Schematics showing typical single-molecule measurement systems for studying myosin-actin interactions. a Dual-beam laser trap assay. Each end of an actin filament is attached to a plastic bead, and the beads are trapped by the focused laser beams. The trapped actin filament is brought to a single myosin molecule attached to a silica bead. b Mechanical measurement system with the use of a thin glass microneedle as a force sensor. A $\mathrm{ZnO}$ crystal whisker is attached to the end of a glass micro-needle (not shown in this schematic). Fluorescently labeled single myosin $\mathrm{S}-1$ is attached to the apex of the $\mathrm{ZnO}$ crystal whisker via a specific binding of biotin-streptavidin. The tip is brought onto actin bundles formed by $\alpha$-actinin. The displacements of the S-1 resulting from

and torsional rigidity of a single actin filament was determined using a glass micro-needle technique (Kishino and Yanagida 1988) and optical tweezers (Tsuda et al. 1996), respectively. The unbinding force between an actin filament and a single myosin head in the nucleotide-free condition was $\sim 9 \mathrm{pN}$ using an optical tweezers method where an actin filament was pulled approximately along its length (Fig. 6d) (Nishizaka et al. 1995). It was also measured to be $\sim 15 \mathrm{pN}$ with AFM, where a single myosin head was pulled in the direction perpendicular to the actin filament (Fig. 6e) (Nakajima et al. 1997).

As described above, no complete consensus was reached among research groups regarding the myosin step size taken for each ATP hydrolysis, and hence also as to the swinging (d)
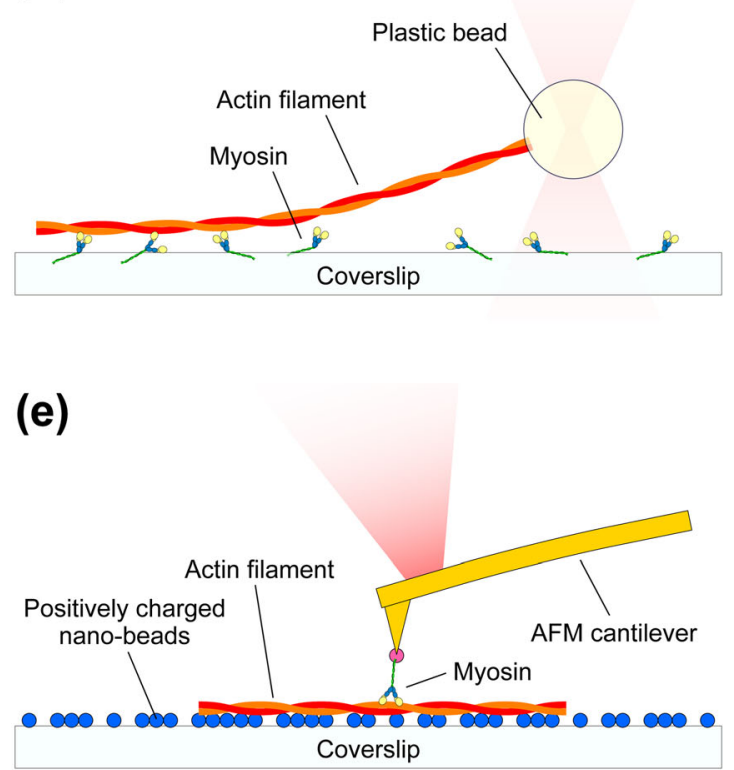

the interaction with actin are monitored by the detection of glass microneedle deflection. $\mathbf{c}$ TIRFM experiment for visualizing individual ATP turnovers by single myosin S-1. The light chain of S-1 molecule is labeled with $\mathrm{Cy} 5$ (indicated by the red star) for the confirmation of whether or not the observed ATP turnovers are those by a single S-1 molecule. Once Cy3-ATP binds to the S-1, the large Brownian motion of the Cy3-ATP stops and therefore its fluorescent spot becomes visible. d Single beam assay for the measurement of unbinding force of a single myosin-actin bond. e AFM measurement of rupture force and rupture distance of a single actin-myosin bond

lever hypothesis. The discrepancy was due partly to a relatively small step size occurring in a short time that was technically difficult to measure.

\section{Myosin V}

The technical difficulty mentioned above was significantly removed by the discovery of M5 that possesses unique structural and biochemical features. M5 is involved in organelle and mRNA transports as well as in membrane trafficking. Its cellular functions and regulations are well described elsewhere (Reck-Peterson et al. 2000; Taylor 2007; Sellers and 
Knight 2007; Sellers and Weisman 2008), so here we focus on its motor mechanism.

\section{Structural and biochemical features of M5}

The most prominent structural feature of two-headed M5 is its neck domain ( $24 \mathrm{~nm})$ (Cheney et al. 1993) which is approximately three-fold longer than that of myosin II ( $~ 8 \mathrm{~nm})$. If M5 really follows the swinging lever-arm hypothesis, a step size markedly larger than that of muscle myosin II should be observed. M5 was biochemically shown to dominate in a high actin-affinity state (ADP-bound state) during its ATPase cycle (De La Cruz et al. 1999), in stark contrast with muscle myosin II where a weak affinity sate (ADP-Pi-bound state) dominates.

\section{Processive movement}

A dual-beam optical trap was used to show that a suspended actin filament successively moved 3-6 times when it interacted with a single M5 molecule attached onto a bead (Mehta et al. 1999). The dwell time depended on the ATP concentration, and therefore, successive step movements differed from the sub-stepping observed previously with myosin II (Kitamura et al. 1999, 2005). This was the first evidence that M5 is a processive motor. Importantly, the step size was $\sim 36 \mathrm{~nm}$, consistent with the swinging lever-arm hypothesis. A much longer processive run (over several microns) of single M5 molecules along surface-immobilized actin filaments was demonstrated with TIRFM (Sakamoto et al. 2000). In a singlebeam optical trap, a trapped bead coated with M5 at low density moved processively with $\sim 36-\mathrm{nm}$ steps along a surface-immobilized actin filament (Rief et al. 2000). The $\sim 36-\mathrm{nm}$ step size corresponds to the head-head distance shown in electron micrographs of M5 with two heads bound to the same actin filament (Walker et al. 2000). From dwell time analyses of the data, the stepping kinetics was shown to be limited by ADP release. In this study, a hand-over-hand model was first proposed, i.e. the two heads alternately switch the leading and trailing positions in every ATPase cycle.

The processivity is consistent with the cellular functions of M5 (e.g., organelle transports). The motility of kinesin, a cargo-carrying microtubule-based processive motor, has been well characterized although its step size is relatively small $(\sim 8 \mathrm{~nm})$ (Vale et al. 1985; Block 2007; Hirokawa et al. 2009). M5 possesses both processivity and a large step size, which were of great advantage in studying details of how M5 moves.

\section{Inhibitory state of M5}

In one of the above studies on the full length of M5 (Sakamoto et al. 2000), the ATP turnover rate was measured biochemically under the same buffer solution conditions (without $\mathrm{Ca}^{2+}$ ) used for the observation of long processive movement. From the maximum actin-activated ATPase rate measured $\left(\sim 1.2 \mathrm{~s}^{-1} / \mathrm{head}\right)$ and the maximum velocity of M5 movement observed with TRIFM $\left(1 \mu \mathrm{m} \mathrm{s}^{-1}\right)$, M5 appeared to travel $\sim 400 \mathrm{~nm}$ per ATPase cycle. This long travel distance implied that a large fraction of M5 (more than $90 \%$ ) would be in a catalytically inhibited or low-activity state. Any M5 not in this state would have a much higher ATPase activity $\left(14 \mathrm{~s}^{-1} /\right.$ head). Interestingly, in previous studies an ATPase rate as high as $14 \mathrm{~s}^{-1}$ /head was reported for single-headed M5 constructs in the absence of $\mathrm{Ca}^{2+}$ (De La Cruz et al. 1999; Trybus et al. 1999) as well as for the full length of M5 in the presence of $\mu \mathrm{M} \mathrm{Ca}^{2+}$ (Nascimento et al. 1996). Later, this mystery of the fast movement $\left(1 \mu \mathrm{m} \mathrm{s}^{-1}\right)$ with a low ATPase activity $\left(\sim 1.2 \mathrm{~s}^{-1} /\right.$ head $)$ was solved by a finding that M5 often takes a folded conformational state in the absence of $\mathrm{Ca}^{2+}$, in which the C-terminal cargo-binding domains bind to the heads, resulting in the inhibition of the ATPase and motor activities (Wang et al. 2004; Krementsov et al. 2004; Li et al. 2006; Liu et al. 2006; Thirumurugan et al. 2006; Olivares et al. 2006). Also, several other myosins such as myosin VII and X are now known to be regulated by head-tail interactions (Yang et al. 2009; Umeki et al. 2009, 2011). Intramolecular folding appears to be a common mechanism to inhibit the activity of motor proteins as a similar conformational transition was shown previously with smooth muscle myosin (Trybus et al. 1982) and kinesin (Hackney et al. 1992). However, a recent study of smooth muscle myosin suggests that an intramolecular interaction between the heads would be a key event in the regulation, rather than a head-tail interaction (Baumann et al. 2012).

\section{Processive hand-over-hand movement}

The 36-nm step size of M5 corresponds to the long pitch pseudo-repeat of the actin helix, suggesting that M5 moves on a plane along an actin filament. This inference was certainly proven to be the case by the observation of the movement of an M5-attached bead on a suspended actin filament. The bead advanced in a left-handed spiral trajectory with one revolution per $2.3 \mu \mathrm{m}$, i.e. only $5.6^{\circ}$ rotation per step (Ali et al. 2002).

The next study to be done was to confirm the tight relationship between the neck length and the step size, using M5 constructs with different neck lengths. It was actually confirmed that the step size is directly proportional to the neck length (Purcell et al. 2002; Sakamoto et al. 2003; Moore et al. 2004; Sakamoto et al. 2005). Thus, the swinging lever-arm hypothesis was nearly secured.

Next, many studies focused on the experimental verification and close inspection of the hand-over-hand movement previously proposed (Rief et al. 2000). To this end, various light microscopy techniques or fluorescent probes were 
introduced to TIRFM: fluorescence imaging with one nanometer accuracy (FIONA) (Yildiz et al. 2003), fluorescence polarization (Forkey et al. 2003), two-color imaging with Qdots (Warshaw et al. 2005), single-molecule high-resolution colocalization of fluorescent dyes (Churchman et al. 2005), a combination of FIONA and fluorescence polarization (Syed et al. 2006), and defocused orientation and position imaging (Toprak et al. 2006). All these studies revealed that in fact M5 walks in a hand-over-hand manner. Not only translational position changes of the two heads but also angular changes of the lever-arm relative to actin were observed where only one of the two lever-arms was labeled with a fluorescent probe. The value of the angle change $\left(\sim 60^{\circ}\right)$ was also consistent with a hand-over-hand movement (Forkey et al. 2003; Syed et al. 2006; Toprak et al. 2006).

These studies supported the hand-over-hand model but only the dwell states were observed when both heads were bound to actin. Therefore, little was known about the fleeting intermediate between the dwell states because the time resolution was low ( $\sim 33 \mathrm{~ms}$ or longer). To increase the time resolution to $1 \mathrm{~ms}$ or less, the following imaging methods were employed: dark field imaging of a 40-nm gold nanoparticle attached to one of the lever-arms (Dunn and Spudich 2007) and recording of light-scattering from a 200-nm M5coated latex bead traveling in an interference fringe pattern (Cappello et al. 2007). Alternatively, the action of walking M5 was slowed down by attaching a large fluorescent microtubule to one of the lever-arms and observed by conventional fluorescence microscopy (Shiroguchi and Kinosita 2007). All three studies revealed that the detached head underwent extensive rotational Brownian motion before landing on a site ahead of the leading head, suggesting that the detached head is unlikely to interact with actin during the Brownian motion. Moreover, it was suggested that the Brownian motion is likely to be biased forward by the orientational change (swing) of the actin-bound leading head, by which the detached head eventually finds an actin ahead of the leading head to become a new leading head. Thus, a clearer picture of the processive hand-over-hand movement was obtained.

\section{Asymmetry of enzymatic kinetics between two heads}

The processive hand-over-hand movement suggests that only the trailing head can detach from actin. If so, there are two possibilities: (Case 1) ADP dissociation followed by ATP binding predominantly occurs at the trailing head, so that the ADP dissociation would be accelerated at the trailing head or retarded at the leading head; (Case 2) the ATP-binding rate would be enhanced at the trailing head or suppressed at the leading head. Between these possibilities, Case 1 appeared more likely as the ATPase rate is limited at the ADP release step. In either case, the kinetics asymmetry between the two heads would be engendered by head-to-head communications through intramolecular strain. This kinetics asymmetry was addressed by solution kinetics studies and optical-trap nanometry studies. In the solution kinetics studies various kinetics constants were compared for S-1 and HMM constructs of M5, although the two heads of M5-HMM do not necessarily interact with the same actin filament in solution (Rosenfeld and Sweeney 2004; Forgacs et al. 2008). These studies suggested retardation of ADP release from the leading head by $\sim 45$-fold (Rosenfeld and Sweeney 2004) or up to 250-fold (Forgacs et al. 2008). The latter study used new fluorescent ADP and ATP analogues (deac-aminoADP and deac-aminoATP) (Webb et al. 2004) whose emission intensity increases by 20 -fold upon binding to the active site of M5 (Forgacs et al. 2006). In the dual-beam trap experiments, the actin filament that was interacting with a surface-attached single-headed M5 molecule was pulled forward or backward to apply a force to the actin-bound head (Purcell et al. 2005; Veigel et al. 2002, 2005). The forward and backward forces were hypothesized to simulate forces experienced by the trailing or the leading head, respectively. Different designs of optical trap nanometry were also used to study the coordination between actin-bound two heads (Uemura et al. 2004; Clemen et al. 2005; Gebhardt et al. 2006; Oguchi et al. 2008). Most of these studies suggested that the intramolecular strain would coordinate two heads by decelerating ADP release from the leading head, whereas some studies suggested accelerated ADP release at the trailing head (Veigel et al. 2002, 2005), as proposed by a study of processive run of M5 perturbed by varying the nucleotide content (Baker et al. 2004).

In two-headed bound M5, the neck of the trailing head takes the arrowhead orientation, whereas the neck of the leading head takes the reverse arrowhead orientation. The leading head conformation must be mechanically distorted because single-headed M5 binds to an actin filament in an arrowhead orientation. In fact, electron micrographs of twoheaded bound M5 showed that the leading neck was curved slightly outwards whereas the trailing neck was straight (Walker et al. 2000; Burgess et al. 2002; Oke et al. 2010). This conformational distortion seems consistent with the suggestion that the coordination of M5 stepping is performed by strain-mediated inhibition of ADP release from the leading head.

Finally, using two-color TIRFM, Sakamoto et al. (2008) simultaneously observed the stepping motion of fluorescently labeled M5 and the binding and dissociation of the fluorescent nucleotide (deac-aminoADP or deacaminoATP). This observation directly revealed the preferential ADP dissociation from the trailing head, which was followed by a 36-nm step triggered by ATP binding. It also revealed that every M5 molecule always keeps at least one nucleotide (ADP in the leading head) during its stepping, even at low ATP concentrations. 
Discrepancies and unsolved questions in myosin V motility

The above description suggests there is now a consensus on how M5 steps. In every ATP hydrolysis cycle, the leading head swings to propel M5 forwards by $\sim 36 \mathrm{~nm}$ (Sakamoto et al. 2008). This large stride is realized by the long lever-arms of M5 (Purcell et al. 2002; Sakamoto et al. 2003; Moore et al. 2004; Sakamoto et al. 2005). The two heads alternately switch between the leading and trailing roles so that M5 walks handover-hand (Yildiz et al. 2003; Forkey et al. 2003; Warshaw et al. 2005; Churchman et al. 2005; Syed et al. 2006; Toprak et al. 2006). This style of movement is made possible by the intramolecular strain-mediated retardation of ADP release from the leading head (Rosenfeld and Sweeney 2004; Purcell et al. 2005; Forgacs et al. 2008; Oguchi et al. 2008; Sakamoto et al. 2008).

Nonetheless, several observations that are difficult to reconcile with this generally agreed view have been reported, mostly from the Yanagida lab. A recombinant two-headed M5 with a single IQ motif on each neck ( $\sim \mathrm{nm}$ long) was reported to move processively with $\sim 36-\mathrm{nm}$ steps (Tanaka et al. 2002). It was claimed that the long-neck domain was not essential for both large step size and processivity of M5 but the motor domain alone determined the processivity and the large step size. It was also reported that single-headed M5 constructs underwent multiple successive large $(\sim 32-\mathrm{nm})$ steps on an actin filament suspended by a dual-beam optical trap (Watanabe et al. 2004). They also underwent directional diffusion on surface-immobilized actin bundles with $\sim 5.5$-nm substeps to develop an average displacement of $\sim 20 \mathrm{~nm}$, which was independent of the neck length (2IQ and 6IQ motifs) (Okada et al. 2007), as previously observed with single-headed myosin II (Kitamura et al. 1999). Likewise, myosin VI with its short neck similar to myosin II, was reported to move with $\sim 36-\mathrm{nm}$ steps (Rock et al. 2001; Nishikawa et al. 2002). However, among these reported irreconcilable conclusions, only the large step size of myosin VI was resolved. The SAHs were found in the proximal tail domain of myosin VI, which contribute to the lever-arm length (Baboolal et al. 2009; Spudich and Sivaramakrishnan 2010; Sweeney and Houdusse 2010b). The other discrepant results are still incomprehensible (Cyranoski 2000; Geeves 2002).

Even if the above general view of how M5 steps is completely right, there remain fundamental questions to solve. How is the chemical energy liberated by ATP hydrolysis used to generate the tension for forward movement? How are the tension generation and lever-arm swing coupled to the ATPase cycle? If they are coupled, which chemical transitions are involved in these mechanical events? These fundamental questions have long been explored in muscle contraction studies and some are considered to be resolved. Are there any inconsistencies when this consensus view is applied to the motility of M5? Numerous single-molecule measurements of M5 stepping have not answered these important questions.

The discrepancies mentioned above, as well as the remaining unsolved fundamental questions, have certainly proven that the level of "directness of measurement" is not yet high enough even with single-molecule optical measurements. Proteins molecules themselves are indeed invisible in these measurements, which can never be overcome even with diffraction-breaking super-resolution fluorescence microscopy (Egner and Hell 2005; Huang et al. 2009).

\section{HS-AFM}

HS-AFM has overcome this technical limitation. Biological molecules can be directly observed at sub-molecular spatial and sub-100 ms temporal resolution without disturbing their function. In this section, we briefly summarize the principle of AFM and the current state of HS-AFM performance (Fig. 7). Techniques involved in the HS-AFM instrument are well described elsewhere (Ando et al. 2001; Ando et al. 2008; Ando 2012). See other reviews for protocols of HS-AFM imaging of protein molecules (Uchihashi et al. 2012) and various application studies of HS-AFM (Ando et al. 2014).

\section{Principles of AFM imaging}

In AFM (Binnig et al. 1986), a sharp tip attached to the free end of a cantilever traces the sample to acquire its surface topography while the sample stage is scanned laterally. AFM can observe any object on a substrate at single-nanometer (or higher) resolution under a wide range of environments (vacuum, air, and liquids). Topography images of biological samples can be acquired under physiological buffer conditions, without fixing, staining and labeling the sample (Engel and Müller 2000; Müller and Dufrêne 2008). Among various imaging modes, the tapping mode (Zhong et al. 1993) is most suited for biological samples. In this mode, the cantilever is excited to oscillate in the Z-direction at or near its first resonant frequency. Because of this oscillation, the cantilever tip is brought intermittently into contact with the sample surface, which can eliminate lateral friction force and hence minimize deformation of fragile samples. The reduction of cantilever oscillation amplitude upon tip-sample contact is measured and then the sample stage is finally moved in the Z-direction to restore the amplitude to its set point using a feedback control. This series of operations is repeated many times for different sample surface points during lateral scanning of the sample stage. Consequently, the 3D movement of the sample stage approximately traces the sample surface, and hence, a topography image of the sample can be constructed from the electric signal used to drive the scanner in the Z-direction. 


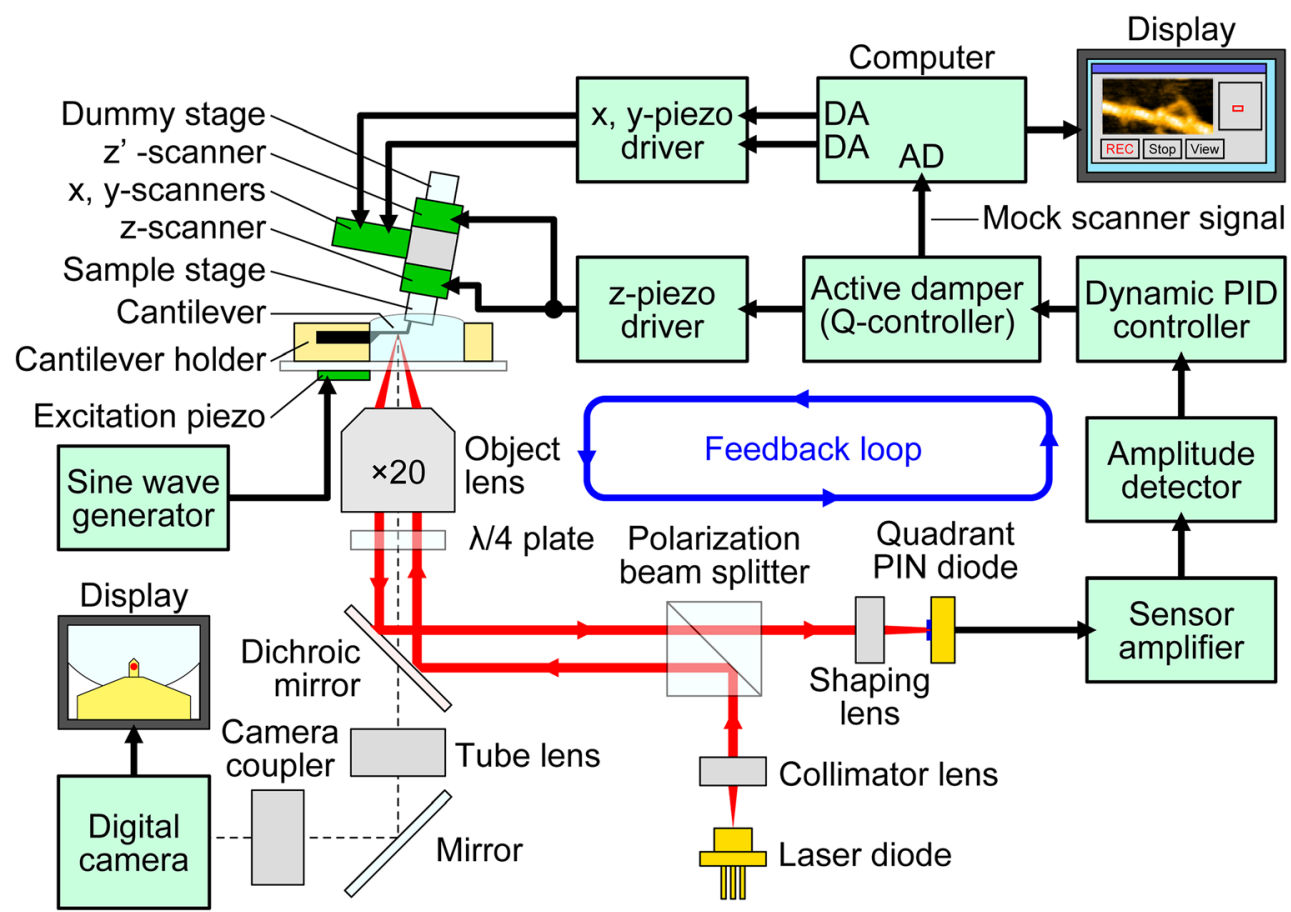

Fig. 7 Schematic of HS-AFM system. The system includes an original inverted optical microscope. The objective lens with a long working distance that is a part of the OBD detector is also used to view the cantilever and sample stage via a digital camera. The glass slide, to which the cantilever holder and the liquid cell are attached, is placed on the optical microscope stage. A cantilever chip is held in the holder so that its tip points upward (opposite to the way in conventional AFM). The sample stage, attached to the Z-scanner and facing downward, is placed over the cantilever. An incident laser beam passing through the objective lens is focused onto the small cantilever, and the light reflected back from the cantilever is collected and collimated by the same objective lens and guided to the quadrant-cell Si PIN photodiode. The incident and reflected laser beams are separated using the quarter-wavelength $(\lambda / 4)$ plate and the polarization beam splitter. A cantilever immersed in a buffer solution is

\section{Current state of HS-AFM}

With conventional AFM instruments it takes at least $30 \mathrm{~s}$ to capture an image. This slow rate is due mainly to the slow response of the mechanical devices (i.e. cantilevers and Zscanner), whereas many interesting biological phenomena occur much faster. Various efforts have been made to markedly increase the imaging rate of tapping-mode AFM over the past 20 years. Small cantilevers, fast scanners, and fast amplitude detectors were developed to build the first prototypic instruments (Viani et al. 2000; Ando et al. 2001). The improvement of these devices and creation of new techniques for vibration damping and fast feedback control produced the current HS-AFM (Ando et al. 2008). The highest possible imaging rate is a function of the speed of an AFM instrument as well as the imaging conditions and the sample fragility (Uchihashi et al. 2012; Ando et al. 2013). For 50- to 200-nm scan ranges, sufficient to image biological molecules, HSAFM can capture an image within $30-80 \mathrm{~ms}$ without

oscillated with small amplitude (1-2 $\mathrm{nm}$ ) by the excitation with an excitation piezo at the first resonant frequency of the cantilever (0.61.2 $\mathrm{MHz}$ in water). The cantilever oscillation amplitude is maintained constant by feedback control. A counterbalancing method is employed to the Z-scanner to minimize unwanted vibrations; two peazoactuators are attached to the supporting base at its counter sides and simultaneously displaced in the same distance, in the counter directions. The scan signals for the X- and Y-scanner are output from the computer through the DA converters. The output from the active Q-control damper is recorded as the sample height through the AD converter. The active Q-control damper is constructed with an LRC circuit whose transfer function is very similar to that of the Z-scanner. Its electrical output behaves in a way similar to the Z-scanner displacement

disturbing their biological function. The spatial resolution of HS-AFM is now comparable with that of conventional slow AFM even with high-bandwidth detection of the cantilever oscillation amplitude and fast scanning of the sample stage ( $\sim 2 \mathrm{~nm}$ for $\mathrm{XY}$ and $\sim 0.1 \mathrm{~nm}$ for $\mathrm{Z}$ in the best case). HS-AFM has already visualized dynamic biomolecular phenomena (see reviews in Ando 2012; Ando et al. 2013, 2014) and even dynamic phenomena in live cells (Yamashita et al. 2012; Watanabe et al. 2013).

\section{Walking M5 filmed by high-speed AFM}

Substrate surface and observation of unidirectional movement

M5-HMM was directly imaged walking along actin filaments using HS-AFM (Kodera et al. 2010). Here, partially biotinylated actin filaments were immobilized on a surface where 
streptavidin was dispersed at low density on mica-supported planar lipid bilayer containing electrically neutral phospholipids and a biotinylated lipid (Fig. 8a). M5-HMM was never bound directly to the lipid bilayer surface and only interacted with the immobilized actin filaments and moved thereon. The moving M5 was frequently oriented perpendicular to the substrate surface so that its characteristic lateral topography was only occasionally observed. Adding a positively charged lipid to the bilayer solved this problem.

In the presence of 1-2 $\mu \mathrm{M}$ ATP, successive AFM images captured at 7 fps clearly showed M5-HMM moving processively with discrete $\sim 36$-nm steps (Fig. 8 b). The twoheaded bound M5-HMM exhibited unique structural features (Fig. 8c). The junction of the neck with the motor domain appears smooth in the leading head but is always V-shaped in the trailing head because the neck regions emerge from different parts of the motor domain. The short coiled-coil tail was mostly tilted towards the minus end of actin. These structural features, which are totally consistent with an electron microscopic observation (Burgess et al. 2002), can be used to determine the actin polarity when bound M5-HMM is stationary. In addition, the leading head always assumed a straight conformation (slightly curved outwards), which agreed with the straight-neck model proposed for walking M5 (Forkey et al. 2003; Syed et al. 2006; Toprak et al. 2006) but disagreed with the bent-neck model (Walker et al. 2000; Burgess et al. 2002; Snyder et al. 2004; Oke et al. 2010).

The positively charged lipid in the lipid bilayer slightly retarded the translocation velocities. When it was absent, the velocities at various ATP concentrations were similar to those measured by fluorescence microscopy under the same buffer solution condition (Forkey et al. 2003; Baker et al. 2004), indicating negligible effects of the tip-sample interaction on the motor activity. However, molecular behavior during a step could not be resolved as it completed very fast.

\section{Visualization of stepping behavior}

To slow the step, more streptavidin molecules were placed on the lipid bilayer surface as moderate obstacles to the walking of M5-HMM (Fig. 9a), which resolved the stepping process (Fig. 9b). Upon detachment of the trailing head from actin, the leading head appeared to spontaneously rotate from the reverse arrowhead orientation towards the arrowhead orientation. Before completing this rotation, the leading head was halted for a moment by colliding with a streptavidin molecule placed on the way of its natural path. In this halt state, the detached trailing head was displaced forward, positioned most distant from the actin filament (thus, the two heads were aligned nearly straight, pointing opposite directions), and slightly rotated around the neck-neck junction (the second frame in Fig. 9b). Then, the leading head overcame the obstacle and further rotated forwards. Immediately after this the trailing head bound to a forward site of the actin filament to become a new leading head, completing one step. Thus, dynamic processes in the forward step were directly visualized. The observed rotation of the leading head is exactly the swinging lever-arm motion itself initially proposed for the powerstroke of muscle myosin (Huxley 1969). Note that before completing a step the detached trailing head never interacted with actin but passively moved forwards driven by the rotating leading head. This ruled out some models of M5 motility such as the 'inchworm'-like model considered for kinesin (Hua et al. 2002) and the "biased diffusion" model proposed for single-headed myosin II (Kitamura et al. 1999) and single-headed M5 (Okada et al. 2007). M5 strictly followed the hand-over-hand mechanism. In contrast, the other processive myosins VI and X, which function not only as a cargo transporter but also as a structural anchor in cells, were reported to move irregularly, namely inchworm-like stepping, backward stepping, and forward hand-over-hand stepping (Yildiz et al. 2004; Sun et al. 2007; Nishikawa et al. 2010; Ricca and Rock 2010; Sun et al. 2010).

Foot stomp and unwinding of coiled-coil tail

Interestingly, in the two-headed bound state during the dwell, the motor domain of the leading head frequently exhibited brief dissociation and reassociation on the same actin filament, while the molecule remained at approximately the same position on the filament (Fig. 10a). Similarly, the motor domain of the trailing head exhibited a brief translocation by $\sim \pm 5 \mathrm{~nm}$ along the actin filament. We termed these behaviors "foot stomp". The foot stomp was more frequently observed at the leading head than at the trailing head (by a ratio of approximately 3:1). Although not well documented, a foot-stomp-like behavior was previously suggested in fluorescence microscopy observations of walking M5 molecules (Syed et al. 2006; Shiroguchi and Kinosita 2007). More recently, the foot stomp was further confirmed by the observation of walking M5 by high-speed single-molecule polarized fluorescence microscopy (Beausang et al. 2013). Thus, the foot stomp is an inherent behavior of this motor.

The foot stomp at the leading head raises an important question as to the chemo-mechanical coupling in M5. The briefly detached leading head does not carry bound Pi because Pi has already been released from the leading head following its initial attachment to actin (De La Cruz et al. 1999; Olivares et al. 2006). Nevertheless, the detached leading head with its bound ADP reattached to actin while still in the reverse arrowhead orientation. It then swung forward following trailing head detachment. This fact was important because it indicated that tension generation for forward movement can occur without transitioning through the ADP-Pi bound state, i.e. it can occur directly in the ADP-bound state. Thus, the 
Fig. 8 HS-AFM imaging of walking M5-HMM. a Schematic showing assay system used for the HS-AFM imaging. b Successive HS-AFM images showing unidirectional processive movement of M5HMM observed in $1 \mu \mathrm{M}$ ATP. Frame rate, 7 fps; Scan area, $130 \times 65 \mathrm{~nm}^{2}$ with $80 \times 40$ pixels; scale bar $30 \mathrm{~nm}$. The vertical dashed lines show the centers of mass of the motor domains, and the plus sign indicates the plus end of actin. c Schematic showing two-headed bound M5-HMM to actin. The corresponding AFM movies can be seen at the following web site: http://www. nature.com/nature/journal/v468/ n7320/extref/nature09450-s2. mov

Fig. 9 Stepping behavior of M5HMM visualized by HS-AFM. a Schematic explaining the HSAFM images shown in (b). b Successive HS-AFM images that resolved the stepping behavior of M5-HMM in $1 \mu \mathrm{M}$ ATP. Frame rate, $7 \mathrm{fps}$; scan area, $150 \times 75 \mathrm{~nm}^{2}$ with $80 \times 40$ pixels; scale bar $50 \mathrm{~nm}$. The swinging lever-arm is highlighted with the thin white lines. The vertical dashed lines in (a) and (b) represent the centers of mass of the motor domains, and the plus sign indicates the plus end of actin. The corresponding AFM movies can be seen at the following web site: http://www. nature.com/nature/journal/v468/ n7320/extref/nature09450-s3. mov (a)
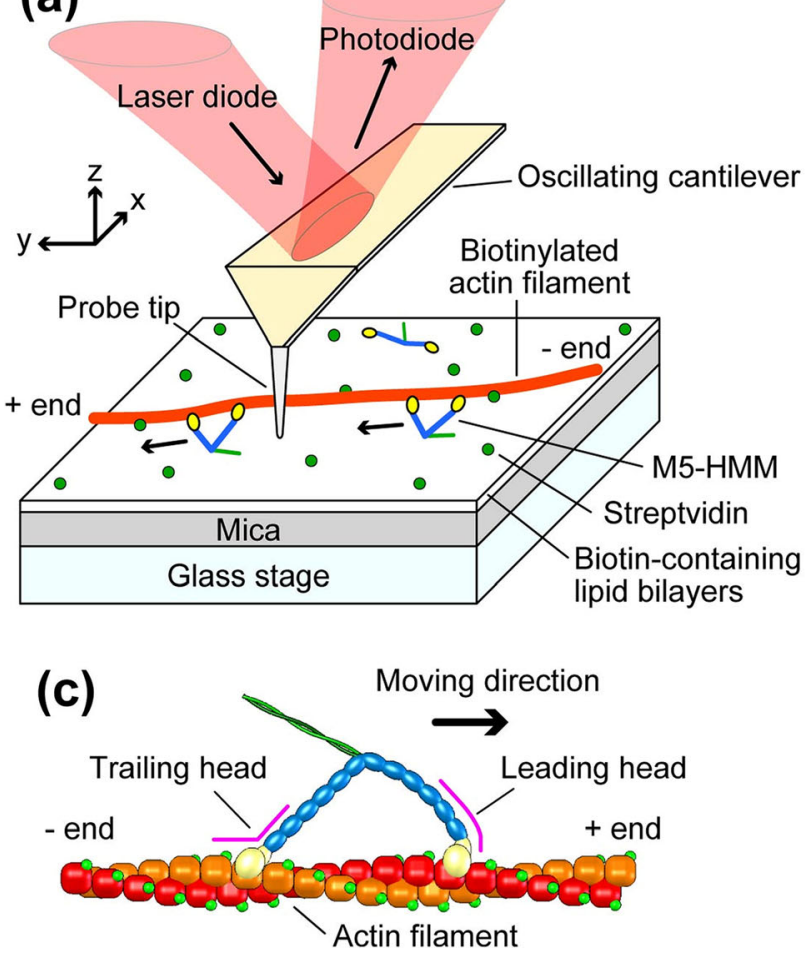

(b)
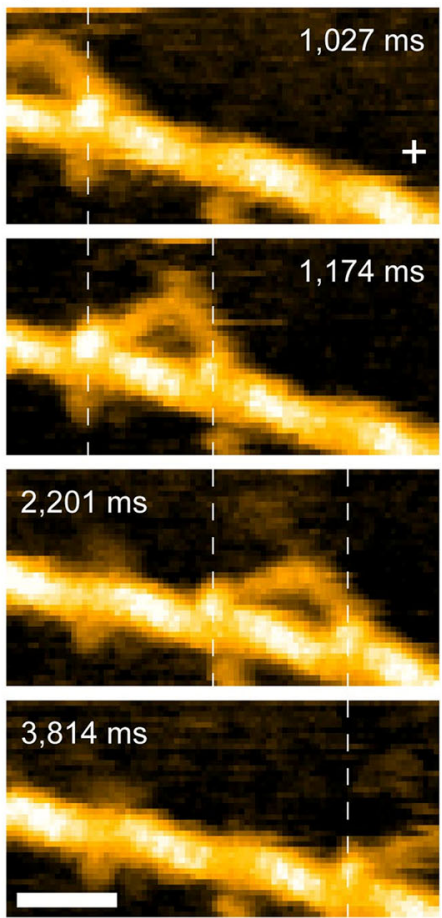

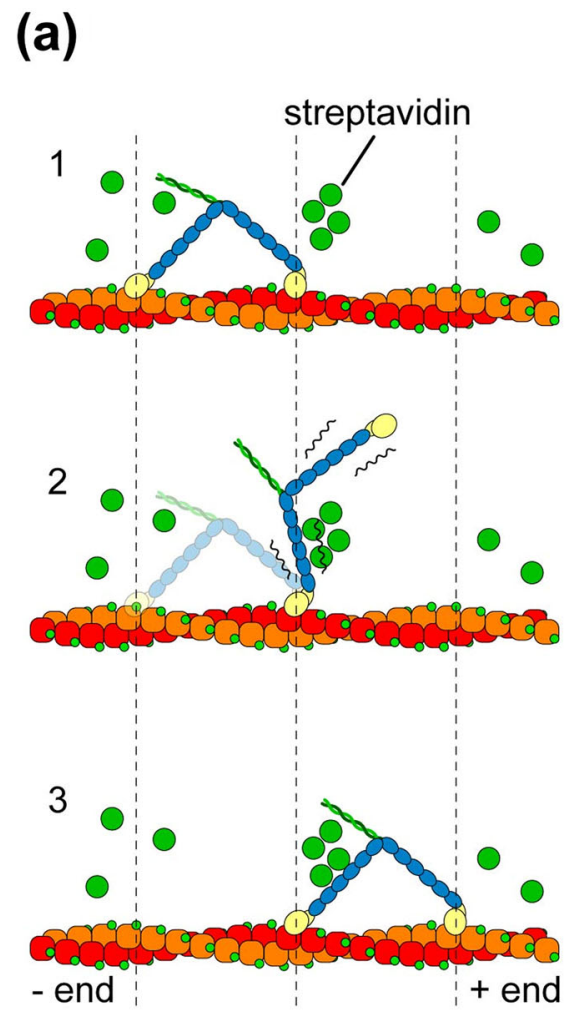

(b)
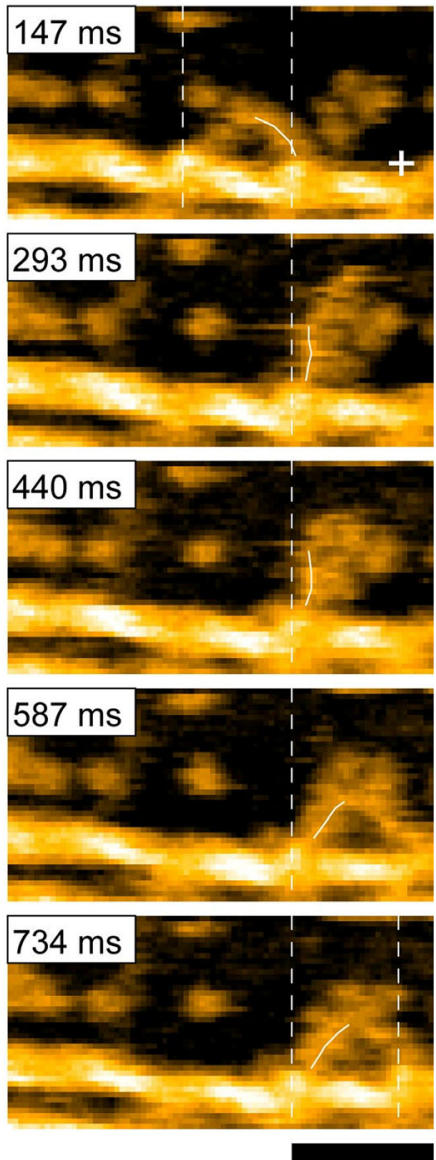
(a)
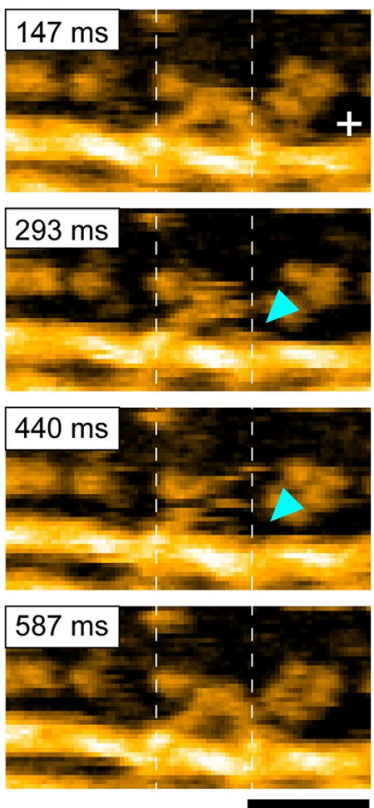

Fig. 10 Unique molecular behaviors of M5-HMM visualized by HSAFM. a Successive HS-AFM images showing a foot stomp event that occurred at the leading-head (ATP, $1 \mu \mathrm{M}$; frame rate, $7 \mathrm{fps}$; scan area, $150 \times 75 \mathrm{~nm}^{2}$ with $80 \times 40$ pixels; scale bar $50 \mathrm{~nm}$ ). The leading-head detachment is marked with the light-blue arrowheads. The corresponding AFM movie can be seen at the following website: http://www.nature. com/nature/journal/v468/n7320/extref/nature09450-s3.mov b HS-AFM images before (upper panel) and after (lower panel) unwinding of coiled-coil tail observed in $50 \mu \mathrm{M}$ ADP. The corresponding AFM movie can be seen at the following website: http://www.nature.com/ nature/journal/v468/n7320/extref/nature09450-s5.mov c HS-AFM

tension generation for forward movement does not seem to require chemical energy be supplied by ATP hydrolysis.

The asymmetry of foot stomp frequency between the two heads suggested their actin-affinity difference, consistent with a biochemically measured result (Olivares et al. 2006). The leading head binds to actin in the unnatural orientation (i.e. reverse arrowhead orientation), and hence, pays an energy cost by distorting the neck conformation, resulting in a lower affinity for actin. The distortion of the leading head is likely to be the source for tension generation for forward movement. This was reinforced by the following observation; when the two-heads were bound to actin in $1 \mathrm{mM}$ ADP, the short coiledcoil tail was sometimes unwound, immediately after which the monomerized leading head rotated towards the arrowhead orientation, similar to the swinging lever-arm motion observed when M5-HMM was walking forwards (Fig. 10b).

Flexibility of neck-motor domain junction

Under nucleotide-free conditions, the leading head frequently exhibited a sharply bent conformation (Fig. 10c) that was never observed in the presence of ATP or in the presence of (c)

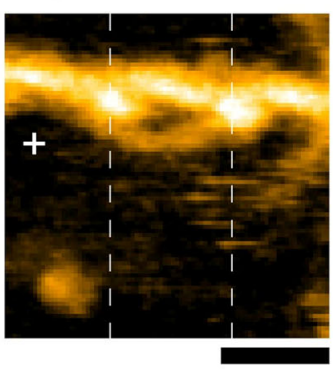

(d)
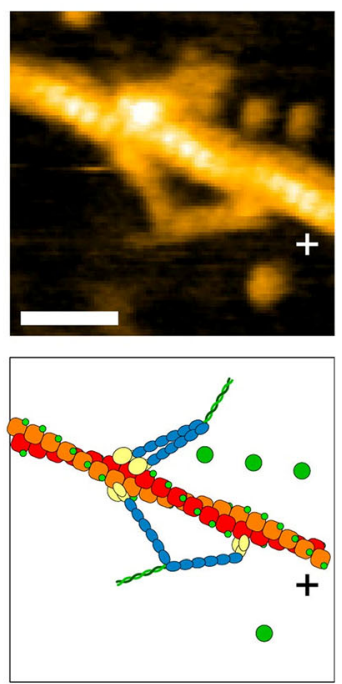

image showing the leading head with a sharply bent conformation observed in the nucleotide-free condition. The corresponding AFM movie can be seen at the following website: http://www.nature.com/ nature/journal/v468/n7320/extref/nature09450-s6.mov d HS-AFM image showing nucleotide-free M5-HMM with heads bound to adjacent actin subunits (upper panel) and its illustration (lower panel). Imaging conditions for (b), (c) and (d) are as followings: frame rate, $3 \mathrm{fps}$; scan area, $100 \times 100 \mathrm{~nm}^{2}$ with $80 \times 80$ pixels; scale bar $30 \mathrm{~nm}$. The vertical dashed lines represent the centers of mass of the motor domains, and the plus signs indicate the plus ends of actin

$1 \mathrm{mM}$ ADP. This bent conformation suggested that the neckmotor domain junction is less flexible in the nucleotide-free head than in the nucleotide-bound head. To examine this issue, the arrowhead orientation angle of single-headed M5 relative to the actin filament was measured under the nucleotide-free and ADP-bound conditions. In nucleotidefree solution, the angle was $\sim 34^{\circ}$, whereas in ADP the angle was distributed widely, peaked at $\sim 29^{\circ}$ and $\sim 51^{\circ}$ in the proportion of approximately 6 to 1 (supplementary data in Kodera et al. 2010), which may be relevant to the two different ADP-bound states in equilibrium as reported for myosin II (Geeves 1989; Geeves and Holmes 1999), M5 (Robblee et al. 2005; Hannemann et al. 2005; Oguchi et al. 2008; Jacobs et al. 2011), and myosin VI (Robblee et al. 2005). Therefore, ADP binding to the head makes the hinge of the neck-motor domain junction flexible. Because of the rigid hinge of the nucleotide-free head, the leading neck in the reverse arrowhead orientation tends to be sharply bent to release the large strain accumulated therein. This rigid hinge was also supported by the observation that both heads are rarely bound to adjacent actin subunits but only in the nucleotide-free condition (Fig. 10d). In the electron micrographs of actomyosin II 
and actomyosin VI complexes, this conformation was frequently seen, suggesting they have rigid hinges (Craig et al. 1980; Nishikawa et al. 2002).

The flexibility of the neck-motor domain junction observed for the actin-bound M5 head with ADP does not seem sufficient to account for the two-headed binding in ADP because the leading head binding in the reverse-arrowhead orientation requires the junction to bend by at least $\sim 60^{\circ}$, even when the contribution of the neck domain's flexibility is considered. This apparently restricted flexibility suggests that the actinunbound head with ADP is more flexible than the actin-bound one. In the other words, actin binding may make the neckmotor domain junction less flexible. This plausible flexibility change seems an excellent strategy for facilitating both actin binding and the generation of enough tension to execute a powerstroke. Very recently, such an actin-binding effect on the flexibility was proposed for muscle myosin (Billington et al. 2014).

\section{Asymmetry of ADP dissociation rate}

The straight and sharply bent conformations of the leading head depend on the presence or absence of bound ADP, respectively, meaning that one can judge whether or not the leading head contains ADP by just looking at its conformation. From the proportion and lifetime of the straight conformation as a function of ADP concentration, the rate constants of ADP binding/dissociation kinetics at the leading head were estimated. The ADP dissociation rate constant was $0.1 \mathrm{~s}^{-1}$, i.e. one ADP is released from the leading head every $10 \mathrm{~s}$, on average. However, M5 walks many steps during $10 \mathrm{~s}$. Thus, from the structural point of view, it was confirmed that the sequential events of ADP release, the subsequent ATP binding and the resulting head dissociation take place solely at the trailing head, which is the basis for the processive hand-overhand movement (Rosenfeld and Sweeney 2004; Purcell et al. 2005; Veigel et al. 2005; Forgacs et al. 2008; Oguchi et al. 2008; Sakamoto et al. 2008).

\section{New questions}

The swinging lever-arm hypothesis is no longer a hypothesis. HS-AFM visualized its occurrence with irrefutable clearness. However, from HS-AFM observations of M5-HMM interacting with actin, questions were raised about the chemo-mechanical coupling in this motor as well as myosin motors in general. The prevailing coupling mechanism had been modeled in the context of swinging lever-arm motion (Fig. 3). In this model, the structural states are putatively tightly coupled to the nucleotide states of the motor domain (Fig. 3). In the M-ADP-Pi state, the head takes the pre- powerstroke conformation caused by recovery stroke of the lever-arm, which facilitates binding of the head to actin in the reverse arrowhead orientation. Coupled to Pi release from the actin-bound head, the intramolecular tension is considered to be generated just once by the pre-powerstroke to postpowerstroke transition. However, the observation with HSAFM of walking M5-HMM showed that the tension responsible for the lever-arm swing can be generated directly by the ADP-bound leading head (after foot stomping). In addition, it was shown that two-headed bound M5-HMM with ADP alone can generate sufficient tension to cause occasional unwinding of the short coiled-coil tail, after which the lever-arm swings. Thus, the recovery stroke or the pre-powerstroke conformation thought to occur uniquely in the ADP-Pi-bound head does not seem necessary for its binding to actin and lever-arm swing. More seriously, the chemical energy liberated by ATP hydrolysis does not seem to be used for the processes of recovery stroke, tension generation, and leverarm swing, suggesting that M5 would step forward without chemical energy input once the trailing head detaches from actin.

This new idea of "no chemical energy usage" in these processes would, however, inevitably lead us to encounter a "perpetuum mobile problem". Even in ADP or in the nucleotide-free condition, foot stomp occurs at both leading and trailing heads of two-headed bound M5-HMM, with a higher frequency in the leading head than in the trailing head. When the trailing head detaches from actin, the molecule takes a forward step by the spontaneous swing of the leading head. On the other hand, when the leading head detaches from actin, the molecule cannot take a backward step because the trailing head is bound to actin in a stable orientation (i.e. the arrowhead orientation). Thus, using only thermal energy, M5 would step forward many times (albeit very slowly), even with less frequent occurrence of foot stomps at the trailing head. This is a forbidden perpetuum motion. However, in this gedankenexperiment, positional fluctuations of the single-headed bound molecule due to the flexible neckmotor domain junction are not considered. Taking this into account, we can expect a backward step after detachment of the leading head from actin, albeit with a low probability. The ratio of foot stomp occurrence between the leading and trailing heads was observed to be $4: 1$ in the presence of $1 \mathrm{mM}$ ADP. However, the foot stomp events at the trailing head mostly occurred as a brief \pm 5 $\mathrm{nm}$ translocation. Therefore, when this brief translocation is omitted and only the events of brief detachment from actin are counted as foot stomp, the ratio of foot stomp occurrence between the two heads becomes much larger. Thus, the principle of detailed balance holds between the forward step after trailing head detachment and the backward step after leading head detachment, resulting in no net movement of the molecule. 
From numerous X-ray crystallography and electron microscopy studies, there is no doubt that myosin changes its conformation upon binding nucleotides and takes a prepowerstroke-like conformation in the ADP-Pi-bound state (Fisher et al. 1995; Smith and Rayment 1996; Dominguez et al. 1998; Houdusse et al. 2000; Burgess et al. 2002; Coureux et al. 2004; Volkmann et al. 2005). These static structures have been used to construct the prevailing model of the chemo-mechanical coupling (Fig. 3) assuming there is a tight one-to-one relationship between the chemical and conformational states. In reality, the relationship is not so tight. Myosin takes two (or multiple) conformations even under a given nucleotide condition. They can go back and forth but the dynamic equilibrium is biased to one side depending on the nucleotide condition. The HS-AFM observations of M5HMM indicates that structural fluctuations indeed exist and therefore two-headed binding occurs even in the ADP-bound and nucleotide free conditions, consistent with other studies (Walker et al. 2000; Rosenfeld and Sweeney 2004; Olivares et al. 2006).

The flexibility of myosin head conformation raises a question whether the chemical energy of ATP hydrolysis is really used to change the conformation. In the prevailing view, the myosin head with a strained (tension-generating) conformation in the pre-powerstroke state is achieved using the chemical energy of ATP, which is finally released by the swinging lever arm motion. However, the pre-powerstroke and postpowerstroke conformations are in dynamic equilibrium with no marked energy barrier. Thus, one can conclude as follows: although the conformational change of myosin head caused by recovery stroke is an effective strategy for facilitating its binding to actin in the reverse arrowhead orientation, the energy required for producing a pre-powerstroke conformation and the tension-generating state is much less than that to be liberated by ATP hydrolysis. Therefore, the conformational change can occur thermally without chemical energy input. At first glance this new view seems inconsistent with the recent report (Shiroguchi et al. 2011) in which the energy barrier between the pre-powerstroke state conformation (formed immediately after ATP addition) and post-powerstroke conformation (formed in the absence of nucleotides) was estimated to be $5.2 k_{\mathrm{B}} T$ ( $k_{\mathrm{B}}$, Bolzmann constant; $T$, room temperature in Kelvin). For this estimation, they monitored the angular distributions of a large bead duplex attached to the motor domain of a single-headed M5 construct, while the molecule was immobilized to a surface of a $50-\mathrm{nm}$ bead by the neck region. This estimated energy barrier seems too large to be achieved thermally. However, the angle distribution measurement did not include the contribution of the flexible neck region to the angle fluctuations, because the neck region was attached to a surface. If it was included, the energy barrier could be smaller. Moreover, the observed angular distributions only reflect projections in the image plane. Therefore, the distributions are underestimated and hence the energy barrier is overestimated. In fact, from solution kinetics measurements of muscle myosin ATPase reaction, the free energy change in the recovery stroke-involved transition from M-ATP to M-ADP-Pi is estimated to be $2 k_{\mathrm{B}} \mathrm{T}$ (Howard 2001). Moreover, the attachment of a large bead duplex to the head would significantly impede the otherwise frequent thermal transitions between the pre-powerstroke and post-powerstroke states.

Let us perform another gedankenexperiment in the presence of ADP alone. Suppose each head of M5 is labeled with a chromophore. Upon light absorption, the chromophore on the bound head will alter its conformation so that it dissociates from actin. When a light pulse is illuminated only at the trailing head of the bound M5, it spontaneously steps forward after detachment of the trailing head. By repeated light illumination only at the trailing head, the molecule would move for a long distance unidirectionally.

It seems plausible that a large fraction of the chemical energy liberated by ATP hydrolysis is used elsewhere and could be used to detach the actin-bound nucleotide-free head from actin. How much energy is required? As mentioned in "Single-molecule measurements", the unbinding (rupture) force was previously measured to be $\sim 15 \mathrm{pN}$ by AFM (Nakajima et al. 1997). The rupture distance was also estimated to be $1.7-2.5 \mathrm{~nm}$ in this study. This distance is unusually long compared to $0.23 \mathrm{~nm}$ for antigen-antibody (Hinterdorfer et al. 1996), $0.15-0.3 \mathrm{~nm}$ for streptavidinbiotin (Yuan et al. 2000), and $0.05-0.3 \mathrm{~nm}$ for $\alpha$-actininactin (Miyata et al. 1996). However, it is consistent with the fact that actin-myosin interfaces contain several bonds formed by flexible loops (Milligan 1996; Kabsch et al. 1990). From the values of rupture force and rupture distance, the energy required to rupture the actin-myosin rigor bond is $6-9 k_{\mathrm{B}} T$, which is $30-45 \%$ of the chemical energy of ATP hydrolysis $\left(\sim 20 k_{\mathrm{B}} T\right)$. This mechanically estimated value is approximately consistent with the values of 9 and $10 k_{\mathrm{B}} T$ estimated biochemically for the free energy changes in the dissociation $\mathrm{A}-\mathrm{M} \rightarrow \mathrm{A}+\mathrm{M}$ and the ATPinduced dissociation $\mathrm{A}-\mathrm{M}+\mathrm{T} \rightarrow \mathrm{A}+\mathrm{M}-\mathrm{T}$, respectively (Howard 2001). One may argue against this statement. For instance, ATP is not yet hydrolyzed in the actin-myosin dissociation step, and hence the energy of ATP is not yet liberated in this step. However, the account balance of energy should be considered for the whole cycle of the ATPase reaction. Therefore, unlike chemical cleavage reactions, in the enzyme-catalyzed reaction cycle the ATPbinding energy can be used as a large part of the energy liberated by ATP hydrolysis. The role of the sequential ATPase reaction process after ATP binding and head dissociation from actin would lie in ensuring the unidirectional progression of the mechanical process. For this assurance, only a small fraction of ATP energy, comparable to thermal energy, is sufficient. 


\section{Conclusions}

We have shown how our understanding of the myosin motor mechanism has advanced with increasing level of directness of measurement. When the level of directness is low, arguments can continue without resolution. For example, in the efforts to provide evidence for or against the swinging leverarm hypothesis, experimental data on the structure and dynamic behavior of myosin were obtained separately. Detailed but static structural data have often given the impression that there is a tight relationship between structural and chemical states. The single-molecule biophysical studies of the dynamic behavior of myosin, on the other hand, have revealed a subset of dynamic molecular events that depends on where the optical probe is placed, and hence other subsets are missed. Therefore, constructing models for the myosin motor mechanism on this basis is reminiscent of a jigsaw puzzle with missing pieces that have to be filled by speculation and hypotheses. Directly observing the structural dynamics and dynamic processes of M5-HMM by HS-AFM has significantly reduced the missing parts and revealed a picture that appears similar to but is largely different from that envisaged previously. Surprisingly, it revealed that generation of tension responsible for forward movement and the following leverarm swing can occur without through the ADP-Pi bound state and therefore the mechanical events do not require the input of chemical energy. Thus, the chemical energy of ATP is mainly used for the detachment of the trailing head from actin. Once detached, the forward swing of the leading head and the resulting forward step of the molecule occur spontaneously without chemical energy input. Of course, this issue of energy usage by M5 should be further corroborated in more direct ways. Moreover, this new principle of energy usage needs to be confirmed for other myosin motors as well as for other mechano-enzymes. This important task remains to be accomplished.

Acknowledgments We thank Prof. Cristobal dos Remedios for reading our manuscript and giving valuable comments. This work was supported by JST/CREST (to TA), JST/PRESTO (to NK), and KAKENHI from the Ministry of Education, Culture, Sports, Science and Technology, Japan (\#20221006 and \#24227005 to TA).

Compliance with Ethical Guidelines The authors declare that our experiments described in this manuscript comply with the current laws of Japan where they were performed.

Conflict of interest Noriyuki Kodera and Toshio Ando declare that they have no conflict of interest.

Human and Animal Studies This article does not contain any studies with human or animal subjects performed by the any of the authors.

Open Access This article is distributed under the terms of the Creative Commons Attribution License which permits any use, distribution, and reproduction in any medium, provided the original author(s) and the source are credited.

\section{References}

Ali MY, Uemura S, Adachi K, Itoh H, Kinosita K Jr, Ishiwata S (2002) Myosin V is a left-handed spiral motor on the right-handed actin helix. Nat Struct Biol 9:464-467

Amitani I, Sakamoto T, Ando T (2001) Link between the enzymatic kinetics and mechanical behavior in an actomyosin motor. Biophys J 80:379-397

Ando T, Kodera N, Takai E, Maruyama D, Saito K, Toda A (2001) A high-speed atomic force microscope for studying biological macromolecules. Proc Natl Acad Sci USA 98:12468-12472

Ando T, Uchihashi T, Fukuma T (2008) High-speed atomic force microscopy for nano-visualization of dynamic biomolecular processes. Prog Surf Sci 83:337-437. The HS-AFM technology was established and opened a new avenue to directly visualize biological molecules in action at high spatiotemporal resolution

Ando T (2012) High-speed atomic force microscopy coming of age. Nanotechnology 23:062001

Ando T, Uchihashi T, Kodera N (2013) High-speed AFM and applications to biomolecular systems. Annu Rev Biophys 42:393-414

Ando T, Uchihashi T, Scheuring S (2014) Filming biomoleculear processes by high-speed atomic force microscopy. Chem Rev 114:3120-3188

Ashkin A, Dziedzic JM (1985) Observation of radiation-pressure trapping of particles by alternating light beams. Phys Rev Lett 54:12451248

Baboolal TG, Sakamoto T, Forgacs E, White HD, Jackson SM, Takagi Y, Farrow RE, Molloy JE, Knight PJ, Sellers JR, Peckham M (2009) The SAH domain extends the functional length of the myosin lever. Proc Natl Acad Sci USA 106:22193-22198

Baker JE, Krementsova EB, Kennedy GG, Armstrong A, Trybus KM, Warshaw DM (2004) Myosin V processivity: multiple kinetic pathways for head-to-head coordination. Proc Natl Acad Sci USA 101: 5542-5546

Baumann BAJ, Taylor DW, Huang Z, Tama F, Fagnant PM, Trybus KM, Taylor KA (2012) Phosphorylated smooth muscle heavy meromyosin shows an open conformation linked to activation. J Mol Biol 415:274-287

Beausang JF, Shroder DY, Nelson PC, Goldman YE (2013) Tilting and wobble of myosin $\mathrm{V}$ by high-speed single-molecule polarized fluorescence microscopy. Biophys J 104:1263-1273

Berg JS, Powell BC, Cheney RE (2001) A millennial myosin census. Mol Biol Cell 12:780-794

Billington N, Revill DJ, Burgess SA, Chantler PD, Knight PJ (2014) Flexibility within the heads of muscle myosin-2 molecules. J Mol Biol 426:894-907

Binnig G, Quate CF, Gerber C (1986) Atomic force microscope. Phys Rev Lett 56:930-933

Block SM (2007) Kinesin motor mechanics: binding, stepping, tracking, gating, and limping. Biophys J 92:2986-2995

Bobkov AA, Bobkova EA, Homsher E, Reisler E (1997) Activation of regulated actin by $\mathrm{SH} 1$-modified myosin subfragment 1 . Biochemistry 36:7733-7738

Bookwalter CS, Lord M, Trybus KM (2009) Essential features of the class V myosin from budding yeast for ASH1 mRNA transport. Mol Biol Cell 20:3414-3421

Borejdo J, Putnam S (1977) Polarization of fluorescence from single skinned glycerinated rabbit psoas fibers in rigor and relaxation. Biochim Biophys Acta 459:578-595

Burgess S, Walker M, Wang F, Sellers JR, White HD, Knight PJ, Trinick J (2002) The prepower stroke conformation of myosin V. J Cell Biol 159:983-991 
Bustamante C, Macosko JC, Wuite GJ (2000) Grabbing the cat by the tail: manipulating molecules one by one. Nat Rev Mol Cell Biol 1:130-136

Cappello G, Pierobon P, Symonds C, Busoni L, Gebhardt JC, Rief M, Prost J (2007) Myosin V stepping mechanism. Proc Natl Acad Sci USA 104:15328-15333

Cheney RE, O'Shea MK, Heuser JE, Coelho MV, Wolenski JS, Espreafico EM, Forscher P, Larson RE, Mooseker MS (1993) Brain myosin- $\mathrm{V}$ is a two-headed unconventional myosin with motor activity. Cell 75:13-23

Churchman LS, Okten Z, Rock RS, Dawson JF, Spudich JA (2005) Single molecule high-resolution colocalization of $\mathrm{Cy} 3$ and $\mathrm{Cy} 5$ attached to macromolecules measures intramolecular distances through time. Proc Natl Acad Sci USA 102:1419-1423

Clemen AE, Vilfan M, Jaud J, Zhang J, Barmann M, Rief M (2005) Force-dependent stepping kinetics of myosin-V. Biophys J 88: $4402-4410$

Coluccio LM (2008) Myosins: a superfamily of molecular motors. Vol. 7, proteins and cell regulations series. Springer, Dordrecht

Cooke R (1986) The mechanism of muscle contraction. CRC Crit Rev Biochem 21:53-118

Coureux PD, Sweeney HL, Houdusse A (2004) Three myosin V structures delineate essential features of chemo-mechanical transduction. EMBO J 23:4527-4537

Craig R, Szent-Gyorgyi AG, Beese L, Flicker P, Vibert P, Cohen C (1980) Electron microscopy of thin filaments decorated with a $\mathrm{Ca}^{2+}$-regulated myosin. J Mol Biol 140:35-55

Cyranoski D (2000) Toshio Yanagida: swimming against the tide. Nature 408:764-766

De La Cruz EM, Wells AL, Rosenfeld SS, Ostap EM, Sweeney HL (1999) The kinetic mechanism of myosin V. Proc Natl Acad Sci USA 96:13726-13731

Dominguez R, Freyzon Y, Trybus KM, Cohen C (1998) Crystal structureof a vertebrate smooth muscle myosin motor domain and its complex with the essential light chain: visualization of the prepower stroke state. Cell 94:559-571

dos Remedios CG, Millikan RG, Morales MF (1972a) Polarization of tryptophan fluorescence from single striated muscle fibers. A molecular probe of contractile state. J Gen Physiol 59:103-120

dos Remedios CG, Yount RG, Morales MF (1972b) Individual states in the cycle of muscle contraction. Proc Natl Acad Sci USA 69:2542-2546

Dunn AR, Spudich JA (2007) Dynamics of the unbound head during myosin V processive translocation. Nat Struct Mol Biol 14:246-248

Egner A, Hell SW (2005) Fluorescence microscopy with super-resolved optical sections. Trends Cell Biol 15:207-215

Engel A, Müller DJ (2000) Observing single biomolecules at work with the atomic force microscope. Nat Struct Biol 7:715-718

Finer JT, Simmons RM, Spudich JA (1994) Single myosin molecule mechanics: piconewton forces and nanometre steps. Nature 368: $113-119$

Fisher AJ, Smith CA, Thoden JB, Smith R, Sutoh K, Holden HM, Rayment I (1995) X-ray structures of the myosin motor domain of Dictyostelium discoideum complexed with $\mathrm{MgADPBeF}_{\mathrm{x}}$ and MgADPAlF ${ }_{4}^{-}$. Biochemistry 34:8960-8972

Ford LE, Huxley AF, Simmons RM (1977) Tension responses to sudden length change in stimulated frog muscle fibers near slack length. J Physiol 269:441-515

Foth BJ, Goedecke MC, Soldati D (2006) New insights into myosin evolution and classification. Proc Natl Acad Sci USA 103:3681-3686

Forgacs E, Cartwright S, Kovacs M, Sakamoto T, Sellers JR, Corrie JE, Webb MR, White HD (2006) Kinetic mechanism of myosinV-S1 using a new fluorescent ATP analogue. Biochemistry 45:1303513045

Forgacs E, Cartwright S, Sakamoto T, Sellers JR, Corrie JE, Webb MR, White HD (2008) Kinetics of ADP dissociation from the trail and lead heads of actomyosin V following the power stroke. J Biol Chem 283:766-773
Forkey JN, Quinlan ME, Shaw MA, Corrie JE, Goldman YE (2003) Three-dimensional structural dynamics of myosin $\mathrm{V}$ by single-molecule fluorescence polarization. Nature 422:399-404

Funatsu T, Harada Y, Tokunaga M, Saito K, Yanagida T (1995) Imaging of single fluorescent molecules and individual ATP turnovers by single myosin molecules in aqueous solution. Nature 374:555-559

Gebhardt JC, Clemen AE, Jaud J, Rief M (2006) Myosin-V is a mechanical ratchet. Proc Natl Acad Sci USA 103:8680-8685

Geeves MA (1989) Dynamic interaction between actin and myosin subfragment 1 in the presence of ADP. Biochemistry 28:5864-5871

Geeves MA, Holmes KC (1999) Structural mechanism of muscle contraction. Annu Rev Biochem 68:687-728

Geeves MA (2002) Stretching the lever-arm theory. Nature 415:129-131

Goldman YE (2004) Muscle contraction. In: Hackney DD, Tamanoi F (eds) Energy coupling and molecular motors, vol. 23, the enzyme series. Elsevier, New York, pp 1-53

Guilford WH, Dupuis DE, Kennedy G, Wu J, Patlak JB, Warshaw DM (1997) Smooth muscle and skeletal muscle myosins produce similar unitary forces and displacements in the laser trap. Biophys $\mathrm{J} 72$ : 1006-1021

Hachikubo Y, Ito K, Schiefelbein J, Manstein DJ, Yamamoto K (2007) Enzymatic activity and motility of recombinant arabidopsis myosin XI, MYA1. Plant Cell Physiol 48:886-891

Hackney DD, Levitt JD, Suhan J (1992) Kinesin undergoes a 9 S to $6 \mathrm{~S}$ conformational transition. J Biol Chem 267:8696-8701

Hannemann DE, Cao W, Olivares AO, Robblee JP, De La Cruz EM (2005) Magnesium, ADP, and actin binding linkage of myosin V: evidence for multiple myosin V-ADP and actomyosin V-ADP states. Biochemistry 44:8826-8840

Harada Y, Noguchi A, Kishino A, Yanagida T (1987) Sliding movement of single actin filaments on one-headed myosin filaments. Nature 326:805-808

Harada Y, Sakurada K, Aoki T, Thomas DD, Yanagida T (1990) Mechanochemical coupling in actomyosin energy transduction studied by in vitro movement assay. J Mol Biol 216:49-68

Hartman MA, Finan D, Sivaramakrishnan S, Spudich JA (2011) Principles of unconventional myosin function and targeting. Annu Rev Cell Dev Biol 27:133-155

Higashi-Fujime S (1980) Active movement in vitro of bundle of microfilaments isolated from Nitella cell. J Cell Biol 87:569-578

Higashi-Fujime S (1991) Reconstitution of active movement in vitro based on the actin-myosin interaction. Int Rev Cytol 125:95-138

Higashi-Fujime S, Hozumi T (1996) The mechanism for mechanochemical energy transduction in actin-myosin interaction revealed by in vitro motility assay with ATP analogues. Biochem Biophys Res Commun 221:773-77810

Hill AV (1938) The heat of shortening and the dynamic constants of muscle. Proc R Soc Lond B 126:136-195

Hill AV (1964) The effect of load on the heat of shortening of muscle. Proc R Soc Lond B 159:297-318

Hinterdorfer P, Baumgartner W, Gruber HJ, Schilcher K, Schindler H (1996) Detection and localization of individual antibody-antigen recognition events by atomic force microscopy. Proc Natl Acad Sci USA 93:3477-3481

Hirokawa N, Noda Y, Tanaka Y, Niwa S (2009) Kinesin superfamily motor proteins and intracellular transport. Nat Rev Mol Cell Biol 10: 682-696

Hodges AR, Krementsova EB, Bookwalter CS, Fagnant PM, Sladewski TE, Trybus KM (2012) Tropomyosin is essential for processive movement of a class V myosin from budding yeast. Curr Biol 22: 1410-1416

Homsher E, Wang F, Sellers JR (1992) Factors affecting movement of Factin filaments propelled by skeletal muscle heavy meromyosin. Am J Physiol 262:C714-C723

Houdusse A, Szent-Györgyi AG, Cohen C (2000) Three conformational states of scallop myosin S1. Proc Natl Acad Sci USA 97:11238-11243 
Howard J (2001) ATP hydrolysis. In: Howard J (ed) Mechanics of motor proteins and the cytoskeleton. Signauer, Sunderland, pp 229-244

Hua W, Chung J, Gelles J (2002) Distinguishing inchworm and handover-hand processive kinesin movement by neck rotation measurements. Science 295:844-848

Huang B, Bates M, Zhuang X (2009) Super-resolution fluorescence microscopy. Annu Rev Biochem 78:993-1016

Huxley AF, Niedergerke R (1954) Structural changes in muscle during contraction: interference microscopy of living muscle fibres. Nature 173:971-973

Huxley AF (1957a) Muscle structure and theories of contraction. Prog Biophys Biophys Chem 7:255-318

Huxley AF, Simmons RM (1971) Proposed mechanism of force generation in striated muscle. Nature 233:533-538

Huxley AF (2000) Mechanics and models of the myosin motor. Philos Trans R Soc Lond B 355:433-440

Huxley HE (1953) Electron microscope studies of the organisation of the filaments in striated muscle. Biochim Biophys Acta 12:387-394

Huxley HE, Hanson J (1954) Changes in the cross-striations of muscle during contraction and stretch and their structural interpretation. Nature 173:973-976

Huxley HE (1957b) The double array of filaments in cross-striated muscle. J Biophys Biochem Cytol 3:631-648

Huxley HE (1963) Electron microscope studies on the structure of natural and synthetic protein filaments from striated muscle. J Mol Biol 7: 281-308

Huxley HE (1969) The mechanism of muscular contraction. Science 164: $1356-1365$

Huxley HE, Simmons RM, Faruqi AR, Kress M, Bordas J, Koch MH (1981) Millisecond time-resolved changes in X-ray reflections from contracting muscle during rapid mechanical transients, recorded using synchrotron radiation. Proc Natl Acad Sci USA 78:22972301

Huxley HE, Simmons RM, Faruqi AR, Kress M, Bordas J, Koch MH (1983) Changes in the X-ray reflections from contracting muscle during rapid mechanical transients and their structural implications. J Mol Biol 169:469-506

Huxley HE (2004) Recent X-ray diffraction studies of muscle contraction and their implications. Philos Trans R Soc Lond B 359:1879-1882

Ishijima A, Doi T, Sakurada K, Yanagida T (1991) Sub-piconewton force fluctuations of actomyosin in vitro. Nature 352:301-306

Ishijima A, Kojima H, Higuchi H, Harada Y, Funatsu T, Yanagida T (1996) Multiple- and single-molecule analysis of the actomyosin motor by nanometer-piconewton manipulation with a microneedle: unitary steps and forces. Biophys J 70:383-400

Ishijima A, Kojima H, Funatsu T, Tokunaga M, Higuchi $H$, Tanaka H, Yanagida T (1998) Simultaneous observation of individual ATPase and mechanical events by a single myosin molecule during interaction with actin. Cell 92:161-171

Jacobs DJ, Trivedi D, David C, Yengo CM (2011) Kinetics and thermodyamics of the rate limiting conformational change in the actomyosin V mechanochemical cycle. J Mol Biol 407:716-730

Jontes JD, Wilson-Kubalek EM, Milligan RA (1995) A $32^{\circ}$ tail swing in brush border myosin I on ADP release. Nature 378:751-753

Joo C, Balci H, Ishitsuka Y, Buranachai C, Ha T (2008) Advances in single-molecule fluorescence methods for molecular biology. Annu Rev Biochem 77:51-76

Kabsch W, Mannherz HG, Suck D, Pai EF, Holmes KC (1990) Atomic structure of the actin: DNase I complex. Nature 347:37-44

Katayama E (1989) The effects of various nucleotides on the structure of actin-attached myosin subfragment-1 studied by quick-freeze deepetch electron microscopy. J Biochem 106:751-770

Kimura Y, Toyoshima N, Hirakawa N, Okamoto K, Ishijima A (2003) A kinetic mechanism for the fast movement of Chara myosin. J Mol Biol 328:939-950
Kishino A, Yanagida T (1988) Force measurements by micromanipulation of a single actin filament by glass needles. Nature 334:74-76

Kitamura K, Tokunaga M, Iwane AH, Yanagida T (1999) A single myosin head moves along an actin filament with regular steps of 5.3 nanometres. Nature 397:129-134

Kitamura K, Tokunaga M, Esaki S, Hikikoshi Iwane A, Yanagida T (2005) Mechanism of muscle contraction based on stochastic properties of single actomyosin motors observed in vitro. Biophysics 1 : $1-19$

Knight PJ, Thirumurugan K, Xu Y, Wang F, Kalverda AP, Stafford WF III, Sellers JR, Peckham M (2005) The predicted coiled-coil domain of myosin 10 forms a novel elongated domain that lengthens the head. J Biol Chem 280:34702-34708

Kodera N, Yamamoto D, Ishikawa R, Ando T (2010) Video imaging of walking myosin $\mathrm{V}$ by high-speed atomic force microscopy. Nature 468:72-75. Dynamic behavior of myosin V interacting with actin was visualized by HS-AFM. The molecular movies not only provided corroborative visual evidence for previously speculated or demonstrated molecular behavior (including lever-arm swing), but also revealed more detailed behavior of the molecules that led to a comprehensive understanding of the motor mechanism

Krementsov DN, Krementsova EB, Trybus KM (2004) Myosin V: regulation by calcium, calmodulin, and the tail domain. J Cell Biol 164: 877-886

Kron SJ, Spudich JA (1986) Fluorescent actin filaments move on myosin fixed to a glass surface. Proc Natl Acad Sci USA 83:6272-6276

Kull FJ, Sablin EP, Lau R, Fletterick RJ, Vale RD (1996) Crystal structure of the kinesin motor domain reveals a structural similarity to myosin. Nature 380:550-555

Kuo SC (2001) Using optics to measure biological forces and mechanics. Traffic 2:757-763

Kuroda K, Kamiya N (1975) Active movement of Nitella chloroplasts in vitro. Proc Jpn Acad 51:774-777

Li XD, Jung HS, Mabuchi K, Craig R, Ikebe M (2006) The globular tail domain of myosin Va functions as an inhibitor of the myosin Va motor. J Biol Chem 281:21789-21798

Liu J, Taylor DW, Krementsova EB, Trybus KM, Taylor KA (2006) Three-dimensional structure of the myosin $\mathrm{V}$ inhibited state by cryoelectron tomography. Nature 442:208-211

Lorenz M, Holmes KC (2010) The actin-myosin interface. Proc Natl Acad Sci USA 107:12529-12534

Lowey S, Waller GS, Trybus KM (1993) Skeletal muscle myosin light chains are essential for physiological speeds of shortening. Nature 365:454-456

Lymn RW, Taylor EW (1971) Mechanism of adenosine triphosphate hydrolysis by actomyosin. Biochemistry 10:4617-4624

Marriott G, Heidecker M (1996) Light-directed generation of the actinactivated ATPase. Activity of caged heavy meromyosin. Biochemistry 35:3170-3174

Maruta H, Korn ED (1977) Purification from Acanthamoeba castellanii of proteins that induce gelation and syneresis of F-actin. J Biol Chem 252:399-402

Mehta AD, Finer JT, Spudich JA (1998) Use of optical traps in singlemolecule study of nonprocessive biological motors. Methods Enzymol 298:436-459

Mehta AD, Rock RS, Rief M, Spudich JA, Mooseker MS, Cheney RE (1999) Myosin- $\mathrm{V}$ is a processive actin-based motor. Nature 400: 590-593

Meyer RK, Aebi U (1990) Bundling of actin filaments by $\alpha$-actinin depends on its molecular length. J Cell Biol 110:2013-2024

Milligan RA (1996) Protein-protein interactions in the rigor actomyosin complex. Proc Natl Acad Sci USA 93:21-26

Miyata H, Yasuda R, Kinoshita K Jr (1996) Strength and lifetime of the bond between actin and skeletal muscle and alpha-actinin studied with an optical trapping technique. Biochim Biophys Acta 1290:83-88 
Molloy JE, Burns JE, Kendrick-Jones J, Tregear RT, White DC (1995) Movement and force produced by a single myosin head. Nature 378: 209-212

Moore JR, Krementsova EB, Trybus KM, Warshaw DM (2004) Does the myosin V neck region act as a lever? J Muscle Res Cell Motil 25: 29-35

Mornet D, Pantel P, Audemard E, Kassab R (1979) The limited tryptic cleavage of chymotryptic S-1: an approach to the characterization of the actin site in myosin heads. Biochem Biophys Res Commun 89: 925-932

Mornet D, Bertrand R, Pantel P, Audemard E, Kassab R (1981) Structure of the actin-myosin interface. Nature 292:301-306

Müller DJ, Dufrêne YF (2008) Atomic force microscopy as a multifunctional molecular toolbox in nanobiotechnology. Nat Nanotechnol 3: 261-269

Murcia MJ, Garg S, Naumann CA (2007) Single-molecule fluorescence microscopy to determine phospholipid lateral diffusion. Methods Mol Biol 400:277-294

Nagy S, Ricca BL, Norstrom MF, Courson DS, Brawley CM, Smithback PA, Rock RS (2008) A myosin motor that selects bundled actin for motility. Proc Natl Acad Sci USA 105:9616-9620

Nakajima H, Kunioka Y, Nakano K, Shimizu K, Seto M, Ando T (1997) Scanning force microscopy of the interaction events between a single molecule of heavy meromyosin and actin. Biochem Biophys Res Commun 234:178-182

Nascimento AA, Cheney RE, Tauhata SB, Larson RE, Mooseker MS (1996) Enzymatic characterization and functional domain mapping of brain myosin-V. J Biol Chem 271:17561-17569

Neuman KC, Block SM (2004) Optical trapping. Rev Sci Instrum 75: 2787-2809

Nihei T, Mendelson RA, Botts J (1974) Use of fluorescence polarization to observe changes in attitude of S-1 moieties in muscle fibers. Biophys J 4:236-242

Nishikawa S, Homma K, Komori Y, Iwaki M, Wazawa T, Hikikoshi Iwane A, Saito J, Ikebe R, Katayama E, Yanagida T, Ikebe M (2002) Class VI myosin moves processively along actin filaments backward with large steps. Biochem Biophys Res Commun 290:311317

Nishikawa S, Arimoto I, Ikezaki K, Sugawa M, Ueno H, Komori T, Iwane AH, Yanagida T (2010) Switch between large hand-overhand and small inchworm-like steps in myosin VI. Cell 142:879888

Nishizaka T, Miyata H, Yoshikawa H, Ishiwata S, Kinosita K Jr (1995) Unbinding force of a single motor molecule of muscle measured using optical tweezers. Nature 377:251-254

Odronitz F, Kollmar M (2007) Drawing the tree of eukaryotic life based on the analysis of 2,269 manually annotated myosins from 328 species. Genome Biol 8:R196

Oguchi Y, Mikhailenko SV, Ohki T, Olivares AO, De La Cruz EM, Ishiwata S (2008) Load-dependent ADP binding to myosins V and VI: implications for subunit coordination and function. Proc Natl Acad Sci USA 105:7714-7719

Okada T, Tanaka H, Iwane AH, Kitamura K, Ikebe M, Yanagida T (2007) The diffusive search mechanism of processive myosin class- $\mathrm{V}$ motor involves directional steps along actin subunits. Biochem Biophys Res Commun 354:379-384

Oke OA, Burgess SA, Forgacs E, Knight PJ, Sakamoto T, Sellers JR, White H, Trinick J (2010) Influence of lever structure on myosin 5a walking. Proc Natl Acad Sci USA 107:2509-2514

Olivares AO, Chang W, Mooseker MS, Hackney DD, De La Cruz EM (2006) The tail domain of myosin Va modulates actin binding to one head. J Biol Chem 281:31326-31336

Oplatka A, Tirosh R (1973) Active streaming in actomyosin solutions. Biochim Biophys Acta 305:684-688

Park H, Ramamurthy B, Travaglia M, Safer D, Chen L-Q, Franzini-Armstrong C, Selvin PR, Sweeney HL (2006) Full- length myosin VI dimerizes and moves processively along actin filaments upon monomer clustering. Mol Cell 21:331336

Park H, Toprak E, Selvin PR (2007) Single-molecule fluorescence to study molecular motors. Q Rev Biophys 40:87-111

Pate E, Franks-Skiba K, White H, Cooke R (1993a) The use of differing nucleotides to investigate cross-bridge kinetics. J Biol Chem 268: 10046-10053

Pate E, White H, Cooke R (1993b) Determination of the myosin step size from mechanical and kinetic data. Proc Natl Acad Sci USA 90: 2451-2455

Pecham M (2011) Coiled coils and SAH domains in cytoskeletal molecular motors. Biochem Soc Trans 39:1142-1148

Pollard TD, Korn ED (1973) Acanthamoeba myosin. I isolation from Acanthamoeba castellanii of an enzyme similar to muscle myosin. $\mathrm{J}$ Biol Chem 248:4682-4690

Pollard TD, Bhandari D, Maupin P, Wachsstock D, Weeds AG, Zot HG (1993) Direct visualization by electron microscopy of the weakly bound intermediates in the actomyosin adenosine triphosphatase cycle. Biophys J 64:454-471

Purcell TJ, Morris C, Spudich JA, Sweeney HL (2002) Role of the leverarm in the processive stepping of myosin V. Proc Natl Acad Sci USA 99:14159-14164

Purcell TJ, Sweeney HL, Spudich JA (2005) A force-dependent state controls the coordination of processive myosin V. Proc Natl Acad Sci USA 102:13873-13878

Rayment I, Rypniewski WR, Schmidt-Bäse K, Smith R, Tomchick DR, Benning MM, Winkelmann DA, Wesenberg G, Holden HM (1993a) Three-dimensional structure of myosin subfragment-1: a molecular motor. Science 261:50-58

Rayment I, Holden HM, Whittaker M, Yohn CB, Lorenz M, Holmes KC, Milligan RA (1993b) Structure of the actin-myosin complex and its implications for muscle contraction. Science 261:58-65

Reck-Peterson SL, Provance DW Jr, Mooseker MS, Mercer JA (2000) Class V myosins. Biochim Biophys Acta 1496:36-51

Reck-Peterson SL, Tyska MJ, Novick PJ, Mooseker MS (2001) The yeast class V myosins, Myo2p and Myo4p, are nonprocessive actin-based motors. J Cell Biol 153:1121-1126

Regnier M, Lee DM, Homsher E (1998) ATP analogs and muscle contraction: mechanics and kinetics of nucleoside triphosphate binding and hydrolysis. Biophys J 74:3044-3058

Ricca BL, Rock RS (2010) The stepping pattern of myosin X is adapted for processive motility on bundled actin. Biophys J 99:1818-1826

Richards TA, Cavalier-Smith T (2005) Myosin domain evolution and the primary divergence of eukaryotes. Nature 436:1113-1118

Rief M, Rock RS, Mehta AD, Mooseker MS, Cheney RE, Spudich JA (2000) Myosin-V stepping kinetics: a molecular model for processivity. Proc Natl Acad Sci USA 97:9482-9486

Robblee JP, Cao W, Henn A, Hannemann DE, De La Cruz EM (2005) Thermodynamics of nucleotide binding to actomyosin V and VI: a positive heat capacity change accompanies strong ADP binding. Biochemistry 44:10238-10249

Rock RS, Rice SE, Wells AL, Purcell TJ, Spudich JA, Sweeney HL (2001) Myosin VI is a processive motor with a large step size. Proc Natl Acad Sci USA 98:13655-13659

Root DD, Reisler E (1992) Cooperativity of thiol-modified myosin filaments: ATPase and motility assays of myosin function. Biophys J 63:730-740

Rosenfeld SS, Sweeney HL (2004) A model of myosin V processivity. J Biol Chem 279:40100-40111

Ruppel KM, Spudich JA (1996) Structure-function analysis of the motor domain of myosin. Annu Rev Cell Dev Biol 12:543573

Sakamoto T, Amitani I, Yokota E, Ando T (2000) Direct observation of processive movement by individual myosin $\mathrm{V}$ molecules. Biochem Biophys Res Commun 272:586-590 
Sakamoto T, Wang F, Schmitz S, Xu Y, Xu Q, Molloy JE, Veigel C, Sellers JR (2003) Neck length and processivity of myosin V. J Biol Chem 278:29201-29207

Sakamoto T, Yildiz A, Selvin PR, Sellers JR (2005) Step-size is determined by neck length in myosin V. Biochemistry 44:16203-16210

Sakamoto T, Webb MR, Forgacs E, White HD, Sellers JR (2008) Direct observation of the mechanochemical coupling in myosin Va during processive movement. Nature 455:128-132

Sekine T, Kielly WW (1964) The enzymic properties of N-ethylmaleimide modified myosin. Biochim Biophys Acta 81:336-345

Sellers JR (2000) Myosins: a diverse superfamily. Biochim Biophys Acta 1496:3-22

Sellers JR, Knight PJ (2007) Folding and regulation in myosins II and V. J Muscle Res Cell Motil 28:363-370

Sellers JR, Weisman LS (2008) Myosin V. In: Coluccio (ed) Myosins: a superfamily of molecular motors, vol. 7 , proteins and cell regulations series. Springer, Dordrecht, pp 289-323

Sheetz MP, Spudich JA (1983) Movement of myosin-coated fluorescent beads on actin cables in vitro. Nature 303:31-35. The first simple and robust in vitro actomyosin motility assay system was developed, which opened a new opportunity to study the motor system quantitatively under diverse conditions, and moreover, led to the creation of single-molecule biophysics.

Sheetz MP, Chasan R, Spudich JA (1984) ATP-dependent movement of myosin in vitro: characterization of a quantitative assay. J Cell Biol 99:1867-1871

Shimizu T, Furusawa K, Ohashi S, Toyoshima YY, Okuno M, Malik F, Vale RD (1991) Nucleotide specificity of the enzymatic and motile activities of dynein, kinesin, and heavy meromyosin. J Cell Biol 112:1189-1197

Shiroguchi K, Kinosita K Jr (2007) Myosin V walks by lever action and Brownian motion. Science 316:1208-1212

Shiroguchi K, Chin HF, Hannemann DE, Muneyuki E, De La Cruz EM, Kinosita K Jr (2011) Direct observation of the myosin Va recovery stroke that contributes to unidirectional stepping along actin. PLoS Biol 9:e1001031

Smith CA, Rayment I (1996) X-ray structure of the magnesium (II)-ADP-vanadate complex of the Dictyostelium discoideum myosin motor domain to $1.9 \AA$ A resolution. Biochemistry 35:54045417

Snyder GE, Sakamoto T, Hammer JA 3rd, Sellers JR, Selvin PR (2004) Nanometer localization of single green fluorescent proteins: evidence that myosin $\mathrm{V}$ walks hand-over-hand via telemark configuration. Biophys J 87:1776-1783

Spudich JA, Kron SJ, Sheetz MP (1985) Movement of myosin-coated beads on oriented filaments reconstituted from purified actin. Nature 315:584-586

Spudich JA, Sivaramakrishnan S (2010) Myosin VI: an innovative motor that challenged the swinging lever-arm hypothesis. Nat Rev Mol Cell Biol 11:128-137

Sun Y, Schroeder HW 3rd, Beausang JF, Homma K, Ikebe M, Goldman YE (2007) Myosin VI walks "wiggly" on actin with large and variable tilting. Mol Cell 28:954-964

Sun Y, Sato O, Ruhnow F, Arsenault ME, Ikebe M, Goldman YE (2010) Single-molecule stepping and structural dynamics of myosin X. Nat Struct Mol Biol 17:485-491

Svoboda K, Block SM (1994) Biological applications of optical forces. Annu Rev Biophys Biomol Struct 23:247-285

Sweeney HL, Houdusse A (2010a) Structural and functional insights into the myosin motor mechanism. Annu Rev Biophys 39:539-557

Sweeney HL, Houdusse A (2010b) Myosin VI rewrites the rules for myosin motors. Cell 141:573-582

Syed S, Snyder GE, Franzini-Armstrong C, Selvin PR, Goldman YE (2006) Adaptability of myosin V studied by simultaneous detection of position and orientation. EMBO J 25:1795-1803
Takács B, Billington B, Gyimesi M, Kintses B, Knight PJ, MálnásiCsizmadiaa A, Kovács M (2010) Myosin complexed with ADP and blebbistatin reversibly adopts a conformation resembling the start point of the working stroke. Proc Natl Acad Sci USA 107:6799-6804

Takano M, Terada TP, Sasai M (2010) Unidirectional Brownian motion observed in an in silico single molecule experiment of an actomyosin motor. Proc Natl Acad Sci USA 107:7769-7774

Tanaka H, Ishijima A, Honda M, Saito K, Yanagida T (1998) Orientation dependence of displacements by a single one-headed myosin relative to the actin filament. Biophys J 75:1886-1894

Tanaka H, Homma K, Iwane AH, Katayama E, Ikebe R, Saito J, Yanagida T, Ikebe M (2002) The motor domain determines the large step of myosin-V. Nature 415:192-195

Taylor KA (2007) Regulation and recycling of myosin V. Curr Opin Cell Biol 19:67-74

Terada TP, Sasai M, Yomo T (2002) Conformational change of the actomyosin complex drives the multiple stepping movement. Proc Natl Acad Sci USA 99:9202-9206

Thirumurugan K, Sakamoto T, Hammer JA 3rd, Sellers JR, Knight PJ (2006) The cargo-binding domain regulates structure and activity of myosin 5. Nature 442:212-215

Thomas DD, Cooke R (1980) Orientation of spin-labeled myosin heads in glycerinated muscle fibers. Biophys J 32:891-906

Thompson RF, Langford GM (2002) Myosin superfamily evolutionary history. Anat Rec 268:276-289

Tokuo H, Mabuchi K, Ikebe M (2007) The motor activity of myosin-X promotes actin fiber convergence at the cell periphery to initiate filopodia formation. J Cell Biol 179:229-238

Tominaga M, Kojima H, Yokota E, Orii H, Nakamori R, Katayama E, Anson M, Shimmen T, Oiwa K (2003) Higher plant myosin XI moves processively on actin with $35 \mathrm{~nm}$ steps at high velocity. EMBO J 22:1263-1272

Toprak E, Enderlein J, Syed S, McKinney SA, Petschek RG, Ha T, Goldman YE, Selvin PR (2006) Defocused orientation and position imaging (DOPI) of myosin V. Proc Natl Acad Sci USA 103:64956499

Toyoshima YY, Kron SJ, McNally EM, Niebling KR, Toyoshima C, Spudich JA (1987) Myosin subfragment-1 is sufficient to move actin filaments in vitro. Nature 328:536-539

Toyoshima YY, Kron SJ, Spudich JA (1990) The myosin step size: measurement of the unit displacement per ATP hydrolyzed in an in vitro assay. Proc Natl Acad Sci USA 87:7130-7134

Trybus KM, Huiatt TW, Lowey S (1982) A bent monomeric conformation of myosin from smooth muscle. Proc Natl Acad Sci USA 79: $6151-6155$

Trybus KM, Krementsova E, Freyzon Y (1999) Kinetic characterization of a monomeric unconventional myosin V construct. J Biol Chem 274:27448-27456

Tsiavaliaris G, Fujita-Becker S, Manstein DJ (2004) Molecular engineering of a backwards-moving myosin motor. Nature 427:558-561

Tsuda Y, Yasutake H, Ishijima A, Yanagida T (1996) Torsional rigidity of single actin filaments and actin-actin bond breaking force under torsion measured directly by in vitro micromanipulation. Proc Natl Acad Sci USA 93:12937-12942

Uchihashi T, Kodera N, Ando T (2012) Guide to video recording of structure dynamics and dynamic processes of proteins by high-speed atomic force microscopy. Nat Protoc 7:1193-1206

Uemura S, Higuchi H, Olivares AO, De La Cruz EM, Ishiwata S (2004) Mechanochemical coupling of two substeps in a single myosin $\mathrm{V}$ motor. Nat Struct Mol Biol 11:877-883

Umeki N, Jung HS, Watanabe S, Sakai T, Li XD, Ikebe R, Craig R, Ikebe M (2009) The tail binds to the head-neck domain, inhibiting ATPase activity of myosin VIIA. Proc Natl Acad Sci USA 106:8483-8488

Umeki N, Jung HS, Sakai T, Sato O, Ikebe R, Ikebe M (2011) Phospholipid-dependent regulation of the motor activity of myosin X. Nat Struct Mol Biol 18:783-788 
Umemoto S, Bengur AR, Sellers JR (1989) Effect of multiple phosphorylations of smooth muscle and cytoplasmic myosins on movement in an in vitro motility assay. J Biol Chem 264:1431-1436

Uyeda TQ, Warrick HM, Kron SJ, Spudich JA (1991) Quantized velocities at low myosin densities in an in vitro motility assay. Nature 352: 307-311

Uyeda TQ, Ruppel KM, Spudich JA (1994) Enzymatic activities correlate with chimaeric substitutions at the actin-binding face of myosin. Nature 368:567-569

Vale RD, Szent-Gyorgyi AG, Sheetz MP (1984) Movement of scallop myosin on Nitella actin filaments: regulation by calcium. Proc Natl Acad Sci USA 81:6775-6778

Vale RD, Reese TS, Sheetz MP (1985) Identification of a novel forcegenerating protein, kinesin, involved in microtubule-based motility. Cell 42:39-50

Veigel C, Coluccio LM, Jontes JD, Sparrow JC, Milligan RA, Molloy JE (1999) The motor protein myosin-I produces its working stroke in two steps. Nature 398:530-533

Veigel C, Wang F, Bartoo ML, Sellers JR, Molloy JE (2002) The gated gait of the processive molecular motor, myosin V. Nat Cell Biol 4:59-65

Veigel C, Schmitz S, Wang F, Sellers JR (2005) Load-dependent kinetics of myosin- $\mathrm{V}$ can explain its high processivity. Nat Cell Biol 7:861-869

Viani MB, Pietrasanta LI, Thompson JB, Chand A, Gebeshuber IC, Kindt JH, Richter M, Hansma HG, Hansma PK (2000) Probing proteinprotein interactions in real time. Nat Struct Biol 7:644-647

Volkmann N, Liu H, Hazelwood L, Krementsova EB, Lowey S, Trybus KM, Hanein D (2005) The structural basis of myosin V processive movement as revealed by electron cryomicroscopy. Mol Cell 19:595-605

Walker ML, Trinick J (1988) Visualization of domains in native and nucleotide-trapped myosin heads by negative staining. J Muscle Res Cell Motil 9:359-366

Walker ML, Burgess SA, Sellers JR, Wang F, Hammer JA 3rd, Trinick J, Knight PJ (2000) Two-headed binding of a processive myosin to Factin. Nature 405:804-807

Wang F, Thirumurugan K, Stafford WF, Hammer JA, Knight PJ, Sellers JR (2004) Regulated conformation of myosin V. J Biol Chem 279: 2333-2336

Wang MD (1999) Manipulation of single molecules in biology. Curr Opin Biotechnol 10:81-86

Warshaw DM, Kennedy GG, Work SS, Krementsova EB, Beck S, Trybus KM (2005) Differential labeling of myosin V heads with quantum dots allows direct visualization of hand-over-hand processivity. Biophys J 88:L30-L32

Watanabe H, Uchihashi T, Kobashi T, Shibata M, Nishiyama J, Yasuda R, Ando T (2013) Wide-area scanner for high-speed atomic force microscopy. Rev Sci Instrum 84:053702
Watanabe T, Tanaka H, Iwane AH, Maki-Yonekura S, Homma K, Inoue A, Ikebe R, Yanagida T, Ikebe M (2004) A one-headed class V myosin molecule develops multiple large (approximately 32-nm) steps successively. Proc Natl Acad Sci USA 101:96309635

Webb MR, Reid GP, Munasinghe VR, Corrie JE (2004) A series of related nucleotide analogues that aids optimization of fluorescence signals in probing the mechanism of P-loop ATPases, such as actomyosin. Biochemistry 43:14463-14471

Wells AL, Lin AW, Chen LQ, Safer D, Cain SM, Hasson T, Carragher BO, Milligan RA, Sweeney HL (1999) Myosin VI is an actin-based motor that moves backwards. Nature 401:505-508

Yamashita H, Taoka A, Uchihashi T, Asano T, Ando T, Fukumori Y (2012) Single-molecule imaging on living bacterial cell surface by high-speed AFM. J Mol Biol 422:300-309

Yanagida T, Nakase M, Nishiyama K, Oosawa F (1984) Direct observation of motion of single F-actin filaments in the presence of myosin. Nature 307:58-60

Yanagida T, Arata T, Oosawa F (1985) Sliding distance of actin filament induced by a myosin crossbridge during one ATP hydrolysis cycle. Nature 316:366-369

Yang Y, Kovács M, Sakamoto T, Zhang F, Kiehart DP, Sellers JR (2006) Dimerized Drosophila myosin VIIa: a processive motor. Proc Natl Acad Sci USA 103:5746-5751

Yang Y, Baboolal TG, Siththanandan V, Chen M, Walker ML, Knight PJ, Peckham M, Sellers JR (2009) A FERM domain autoregulates Drosophila myosin 7a activity. Proc Natl Acad Sci USA 106: 4189-4194

Yano M, Yamamoto Y, Shimizu H (1982) An actomyosin motor. Nature 299:557-559

Yildiz A, Forkey JN, McKinney SA, Ha T, Goldman YE, Selvin PR (2003) Myosin V walks hand-over-hand: single fluorophore imaging with 1.5-nm localization. Science 300:2061-2065

Yildiz A, Park H, Safer D, Yang Z, Chen LQ, Selvin PR, Sweeney HL (2004) Myosin VI steps via a hand-over-hand mechanism with its lever-arm undergoing fluctuations when attached to actin. J Biol Chem 279:37223-37226

Yu C, Feng W, Wei Z, Miyanoiri Y, Wen W, Zhao Y, Zhan M (2009) Myosin VI undergoes cargo-mediated dimerization. Cell 138:537548

Yuan C, Chen A, Kolb P, Moy VT (2000) Energy landscape of streptavidin-biotin complexes measured by atomic force microscopy. Biochemistry 39:10219-10223

Zhong Q, Inniss D, Kjoller K, Elings VB (1993) Fractured polymer silica fiber surface studied by tapping mode atomic-force microscopy. Surf Sci 290:L688-L692 